\title{
Constraining chameleon field driven warm inflation with Planck 2018 data
}

\author{
Haidar Sheikhahmadi ${ }^{1,2, a}{ }^{10}$, Abolhassan Mohammadi $^{3,4, b}$, Ali Aghamohammadi ${ }^{5, c}$, Tiberiu Harko ${ }^{6,7, d}$, \\ Ramón Herrera $^{8, e}$, Christian Corda ${ }^{9,10, f}$, Amare Abebe $^{2, g}$, Khaled Saaidi ${ }^{3, h}$ \\ ${ }^{1}$ School of Astronomy, Institute for Research in Fundamental Sciences (IPM), P. O. Box 19395-5531, Tehran, Iran \\ ${ }^{2}$ Center for Space Research, North-West University, Mafikeng, South Africa \\ ${ }^{3}$ Department of Physics, Faculty of Science, University of Kurdistan, Sanandaj, Iran \\ ${ }^{4}$ Dipartimento di Fisica e Astronomia, Università di Bologna, via Irnerio 46, 40126 Bologna, Italy \\ ${ }^{5}$ Sanandaj Branch, Islamic Azad University, Sanandaj, Iran \\ ${ }^{6}$ Department of Physics, Babes-Bolyai University, Kogalniceanu Street, 400084 Cluj-Napoca, Romania \\ ${ }^{7}$ School of Physics, Sun Yat-Sen University, Xingang Road, Guangzhou 510275, People's Republic of China \\ ${ }^{8}$ Instituto de Física, Pontificia Universidad de Católica de Valparaíso, Casilla 4950, Valparaiso, Chile \\ ${ }^{9}$ Research Institute for Astronomy and Astrophysics of Maragha (RIAAM), P.O. Box 55134-441 Maragha, Iran \\ ${ }^{10}$ International Institute for Applicable Mathematics and Information Sciences (IIAMIS), B.M. Birla Science Centre, Adarsh Nagar, Hyderabad \\ 500463 , India
}

Received: 27 August 2019 / Accepted: 17 December 2019 / Published online: 30 December 2019

(C) The Author(s) 2019

\begin{abstract}
We investigate warm inflationary scenario in which the accelerated expansion of the early Universe is driven by chameleon-like scalar fields. Due to the nonminimal coupling between the scalar field and the matter sector, the energy-momentum tensor of each fluid component is not conserved anymore, and the generalized balance equation is obtained. The new source term in the energy equation can be used to model warm inflation. On the other hand, if the coupling function varies slowly, the model reduces to the standard model used for the description of cold inflation. To test the validity of the warm chameleon inflation model, the results for warm inflationary scenarios are compared with the observational Planck2018 Cosmic Microwave Background data. In this regard, the perturbation parameters such as the amplitude of scalar perturbations, the scalar spectral index and the tensor-to-scalar ratio are derived at the horizon crossing in two approximations, corresponding to the weak and strong dissipative regimes. As a general result it turns out that the theoretical predictions of the chameleon

\footnotetext{
a e-mails: h.sh.ahmadi@gmail.com; h.sheikhahmadi@ipm.ir

b e-mails: abolhassanm@gmail.com; a.mohammadi@uok.ac.ir

c e-mail: a.aqamohamadi@gmail.com

d e-mail: tiberiu.harko@gmail.com

e e-mail: ramon.herrera@pucv.cl

f e-mail: cordac.galilei@gmail.com

g e-mail: amare.abbebe@gmail.com

he-mails: khaledsaeidi@gmail.com; ksaaidi@uok.ac.ir
}

warm inflationary scenario are consistent with the Planck 2018 observations.

\section{Introduction}

Four decades after the introduction of the inflation model, it can now be considered as one of the cornerstones of modern cosmology [1-9]. The main success of this theory goes back to resolving the three main drawbacks of the Standard Big Bang (SBB) theory, namely the flatness, horizon and relic problems [10-15]. In inflation theory we deal with very high energy scales, between $200 \mathrm{GeV}$ and $10^{12} \mathrm{TeV}$ [16], and, by considering the quantum effects inherent to these energies, one can explain the origin of seeds for Large-Scale Structure (LSS) formation, besides the fluctuations generating the Cosmic Microwave Background (CMB) anisotropies [16-25]. In the present work, we will investigate in more detail the relation between quantum fluctuations and classical behaviour at the end of inflation.

Inflationary scenarios usually predict that the power spectrum originated from primary quantum fluctuations should have equal power on all scales, i.e., it should be scale invariant [8,9,26-30]. Although several interesting models have been proposed to explain the origin of the Universe [31-60], inflation, due to its potential for solving the major problems of the SBB theory, has become a crucial part of present day cosmology. To generate an inflationary phase for the very 
early evolution of the Universe, which could explain the symmetries of the cosmological principle, one of the important physical candidates is a scalar field. This type of exotic matter can be described as a fluid with negative pressure that inflation needs to have. In other words, the logarithm of the scalar field potential should move much slower than the kinetic part, leading to the rapid exponential expansion determining the inflationary expansion [1-6].

Despite all of its successes the nature and the origin of the inflationary theory remain a mystery, and it is still a matter of intense scientific investigation $[10,11,16]$. Nevertheless, this is not so surprising for us because inflation happened, as mentioned before, in a very high energy epoch. Up to now, there is no complete or convincing version of the Grand Unification Theory (GUT) that could describe the physics of the very early Universe, and of its beginning. Following [16], and from the observational point of view, we can classify the huge number of different models of inflation into three main categories, with their related sub-categories. These are the single-field inflation [61-74], the multiple-field inflation [75-82] and those models in which the fluid is not described by scalar fields [83-87].

One of the important requirements of the inflationary scenarios is to establish a physically realistic relation between the end of the early accelerated, de Sitter type evolution, and the beginning of the radiation-dominated epoch, which corresponds to the SBB model. Based on this requirement, one can consider two different types of inflation, namely the super-cold and the warm models of inflation [88-100].

Whereas each one of these scenarios has its advantages and drawbacks, in the present work we want to consider both of them in more detail for a single-field model of inflation, based on the chameleon mechanism. But before going through this model, let us explain the properties of super-cold and warm proposals of inflation and their successes and failures. In super-cold models, to establish a relation between the end of inflation and the beginning of the hot big bang model, usually the concept of quantum fluctuations, based on the scalar field oscillations, is considered. In other words, in this approach, one should assume a tachyonic pre-heating phase. Hence, based on this mechanism, the end of inflation is smoothly connected to the radiation-d dominated epoch [75,101-109]. The main problem of this model goes back to the consistency with observations. Unfortunately we know very little, from an observational point of view, about these eras and their evolutions, i.e., the pre-heating and the reheating, and we have no accurate criteria to compare the theoretical results with observations [16]. Recently, different proposals have been proposed to bind existing observational data together to obtain a better estimation of super-cold inflation [21,22,110-139]. Another important constraint originating from this setup, besides the adiabatic initial condition on the $\mathrm{CMB}$, is that the temperature due to reheating has to be larger than the BB Nucleosynthesis (BBN) scales. For more details and for different solutions to overcome this drawback, we refer the reader to Ref. [140], and references therein.

In the present paper we are going to investigate the properties of warm inflation in more detail. A necessary condition for a field theory to produce warm inflation is that the radiation must be in thermal equilibrium. A crucial advantage of this model of inflation is that it does not need any preheating and reheating mechanisms to realize the connection between the end of inflation and the beginning of the radiation era [88-92]. To see how this can be achieved, let us go back to the aforementioned crucial problem of initial quantum fluctuations, related to the super-cold inflation. As opposed to the super-cold model of inflation, in warm inflation one can assume the existence of an interaction between the different components of the initial stages of the Universe as an intrinsic physical property. This assumption plays the role of a master-key for warm inflation. In other words, when the scalar field acquires the zero-point energy of the inflaton, the responsible field in driving inflation, its reaction back on the inflaton can cause the damping of its motion $[92,93,141]$. We must stress that to solve the flatness and horizon problems this reaction should be strong $[92,93]$. Therefore instead of slow-roll mechanisms in warm inflation the concept of overdamped motion can be introduced $[92,93]$.

The prediction of the cosmological quantum perturbations is one of the main achievements of the inflationary scenario. The perturbations are divided into three types: scalar, vector, and tensor perturbations, which evolve independently up to the linear order. The scalar perturbations are known to be the seeds of the LSS of the Universe, while the tensor perturbations are primordial gravitational waves. For the sake of completeness, we recall that the now-famous event GW150914, which is the first direct observation of gravitational waves from a binary black hole merger [142] occurred in the 100th anniversary of Albert Einstein's prediction of gravitational waves [143]. That event was a cornerstone for science in general and for gravitational physics in particular. It indeed gave definitive proof of the existence of gravitational waves, of the existence of black holes having mass greater than 25 solar masses, and of the existence of binary systems of black holes that merge in a time less than the age of the Universe [142].

Such a direct gravitational waves detection represented the starting point of the new era of gravitational waves astronomy. After the event GW150914, the LIGO Scientific Collaboration announced other six new gravitational waves detections, the events GW151226 [144], GW170104 [145], GW170814 [146], GW170817 [147], GW170608 [148] and, recently, GW151012 [149]. All the cited events again are the consequences of binary black hole coalescences, with the sole exception of the event GW170817, which represents the first gravitational waves detection from a neutron star merger 
[147]. Gravitational waves detectors could be, in principle, decisive to confirm the physical consistency of Einstein's general theory of relativity, or, alternatively, to endorse the framework of extended theories of gravity [150,151]. In fact, some differences between the GTR and alternative theories appear in the linearized theory of gravity, and they could be observed through different interferometer response functions [150-152].

As mentioned already, important candidates for the initial seeds of LSS formation are the quantum fluctuations. But the main question is how we can find a mechanism to link quantum fluctuations to these classical seeds, namely the quantum-to-classical transition problem [153-156]. In [156] it was shown that although by considering modes larger than the Hubble length one can treat the fluctuations classically, they are not covariant under canonical transformation $[153,154]$. To solve this problem, a solution was proposed in [157-161], which is based on the neglect of the quantum interaction between macroscopically distinguished events, namely decoherence. Whereas in warm inflation the dissipation behaviour appears as a basic property, the decoherence can automatically generate it. In [92,162-166] different conditions for warm inflation originating from quantum field theory were studied.

The present paper is organized as follows. We first briefly review the chameleon scalar field model in Sect. 2. In particular, we introduce the gravitational action, involving a nonminimal coupling between scalar field and the matter sector, and we write down the generalized Friedmann equations of the theory, as well as the energy balance equation, in which the coupling between scalar field and matter generates a new source term, given by the product of the derivative of the coupling function with respect to the scalar field, and the matter Lagrangian. The chameleon warm inflation model is introduced, and analyzed, in Sect. 3. First, in Sect. 3.1 we review the standard approach to warm inflation, in which a radiation component is present, together with the scalar, during inflation. Due to the interaction between these two components, there is an energy transfer from the scalar field to radiation, due to the presence of a new source term in the conservation equations. The slow-roll parameters and the number of efolds are also obtained. The main theoretical model we investigate in the paper, the chameleon field driven warm inflation model, is presented in detail in Sect. 3.2. In the chameleon warm inflationary model the dissipative term in the conservation equations is automatically generated due to the scalar field-radiation coupling. This also fixes the functional forms of the Hubble function, of the scalar field potential, and of the radiation temperature as functions of the coupling function $f(\phi)$, and, consequently, of the scalar field. Under the assumption that the temperature dependence of the radiation is a power law function of the scalar field, the temperature evolution can be fully determined. Moreover, the scalar spectral index, the amplitude of tensor perturbations, and the tensor spectral index, are also obtained. Usually, the warm inflationary models are investigated in two limiting regimes, corresponding to weak and strong dissipation, respectively. In Sect. 3.3 we investigate the weak dissipative regime, for which the dissipative ratio is much smaller then unity. We perform a detailed physical and numerical analysis of the model in this approximation, by obtaining the slow-roll parameters, the scalar spectral index $n_{s}$, and the tensor to scalar ratio $r$ as functions of the coefficients $n$ and $m$ (characterizing the temperature evolution), and of the number of e-folds. Using the $r-n_{s}$ diagram from the Planck 2018 data, the comparison of the theoretical predictions of the model with the observational data is performed, and the allowable range of parameters is obtained. The evolution of the scalar field potential and of the coupling function $f(\phi)$ is also obtained. The strong dissipative regime is investigated in a similar way in Sect. 3.4, and the allowable ranges of the model parameters are obtained from the comparison with the observational data. The coupling function has an exponential behavior. Finally, in Sect. 4 we discuss and conclude our results.

\section{A brief review of the chameleon model}

As stated earlier, a scalar field-driven early epoch of inflationary expansion is nowadays taken to be as a standard ingredient to describe the expansionary history of the Universe. Less standard, however, is the nature of the inflationary scenario, and of the driving scalar field. One possible candidate suggested in the literature [167-179] is the so-called chameleon scalar field, a light scalar field whose mass depends on the ambient matter density. Originally put forward to overcome the quintessence mechanism drawbacks, and as a dark energy candidate for the late-time cosmic acceleration, recent studies [180-183] have shown that chameleonic inflation is also possible with the appropriate choice of the potential for the scalar field. This leads to a varying mass for the scalar field in which in a dense environment, the scalar field acquires a large mass, which results in short range effects.

In the original chameleon model, the scalar field is coupled to the matter through a conformal factor, $\tilde{g}_{\mu \nu}=e^{\frac{2 \beta \phi}{M_{p}}} g_{\mu \nu}$, which is also the relation between the Jordan and the Einstein frames. However, our case is different in that the scalar field is non-minimally coupled to the matter sector, such that the Lagrangian of the model is given as

$$
S=\int d^{4} x \sqrt{-g}\left(R / 2-\frac{1}{2} \partial_{\mu} \phi \partial^{\mu} \phi-V(\phi)+f(\phi) L_{m}\right) .
$$

Here $g$ is the determinant of the metric tensor $g_{\mu \nu}, R$ the Ricci scalar constructed from the metric, $L_{m}$ is the Lagrangian of the standard matter fields, $V(\phi)$ is the potential of the 
chameleon-like scalar field $\phi$, and $f(\phi)$ is some generic function of the scalar field to be described shortly.

Assuming a spatially flat Friedmann-Robertson-Walker (FRW) metric, the Friedmann equations of the model are given by

$3 H^{2}=\rho_{\phi}+f(\phi) \rho, \quad 2 \dot{H}+3 H^{2}=-p_{\phi}-f(\phi) p$,

where $\rho_{\phi}$ and $p_{\phi}$ are the energy density and pressure of the scalar field, respectively, given by

$\rho_{\phi}=\frac{1}{2} \dot{\phi}^{2}+V(\phi), \quad p_{\phi}=\frac{1}{2} \dot{\phi}^{2}-V(\phi)$,

with an overdot representing the derivative with respect to the cosmic time $t$. Also, taking the variation of the action with respect to the scalar field $\phi$, leads to the equation of motion of the scalar field (the generalized Klein-Gordon equation),

$\ddot{\phi}+3 H \dot{\phi}+V^{\prime}(\phi)=f^{\prime}(\phi) L_{m}$,

where the prime denotes the derivative with respect to the scalar field. The matter Lagrangian in the above equation must be specified in order to close the system of cosmological equation. There are many studies on this topic such as [184-192], where the authors explain that there are two definitions for the Lagrangian of a perfect fluid as $L_{m}^{(1)}=-\rho$ and $L_{m}^{(2)}=p$, respectively. However, in our case, this degeneracy is broken due to the non-minimal interaction of the scalar field and matter, and therefore we follow the approach proposed in [192], where it is shown that only for $L_{m}^{(2)}=p$ one has a geodesic motion for perfect fluids. Thus, in this work, we are going to adopt this Lagrangian for the perfect fluid. Because of the interaction between the scalar field and the matter sector, the conservation equations are generalized, and they are given by

$$
\begin{aligned}
\frac{d}{d t}[f(\phi) \rho]+3 H f(\phi)(\rho+p) & =-\dot{f}(\phi) p, \\
\frac{d}{d t} \rho_{\phi}+3 H\left(\rho_{\phi}+p_{\phi}\right) & =\dot{f}(\phi) p .
\end{aligned}
$$

\section{Chameleon warm inflation}

In this section, we are going to explain the general features of warm inflation, in which the inflaton is described by a chameleon-like scalar field. In warm inflation a dissipation term appears on the right hand side of the conservation equation, which is usually included by hand. However, in the chameleon model of scalar field one could naturally obtain a dissipation term, as in Eq. (4), because of the presence of the interaction between the scalar field, and the matter term in the action. In the following, we first review the warm inflationary scenario, and then we combine the scenario with the chameleon model, where the chameleon scalar field plays the role of the inflaton.

\subsection{Review of warm inflation}

The warm inflationary scenario describes an accelerated expansion phase of the Universe at its earliest times, in which the scalar field is the dominant component. However, its main difference with the cold inflation is that beside a scalar field, there is another component of the cosmological fluid is present, usually taken as radiation. During inflation the scalar field and the matter (radiation) component interact. In such a case, the Friedmann equations are given by

$3 H^{2}=\frac{1}{2} \dot{\phi}^{2}+V(\phi)+\rho_{r}, \quad 2 \dot{H}=-\dot{\phi}^{2}-\frac{4}{3} \rho_{r}$.

Due to this interaction, energy is transferred from the scalar field to the radiation fluid, and this process is described through the following conservation equations,

$$
\begin{aligned}
\dot{\rho}_{r}+3 H\left(\rho_{r}+p_{r}\right) & =\Gamma \dot{\phi}^{2}, \\
\dot{\rho}_{\phi}+3 H\left(\rho_{\phi}+p_{\phi}\right) & =-\Gamma \dot{\phi}^{2} .
\end{aligned}
$$

Here $\Gamma$ is a dissipation coefficient. Equation (7) is usually written in a different way, known as the equation of motion of the scalar field, as

$\ddot{\phi}+3 H(1+Q) \dot{\phi}+V^{\prime}(\phi)=0$,

where the parameter $Q=\Gamma / 3 H$ is the ratio of the radiation production to expansion rate.

In warm inflation, the slow-roll approximations are still at work, i.e., in order to have a quasi-de Sitter expansion, the rate of the Hubble parameter during a Hubble time is assumed to be small, a condition that is imposed via the first slow-roll parameter

$\varepsilon_{1}=-\frac{\dot{H}}{H^{2}}$.

The energy density of the scalar field dominates over the radiation energy density, and also the kinetic term of the scalar field is negligible w.r.t its potential, i.e. $\rho_{\phi} \gg \rho_{r}$ and $\rho_{\phi} \simeq V(\phi)$. In addition to the assumptions that we also have in cold inflation, in warm inflationary scenario it is supposed that the radiation production is quasi-stable during inflation, so that $\dot{\rho}_{r} \ll H \rho_{r}$ and $\dot{\rho}_{r} \ll \Gamma \dot{\phi}^{2}$. Then, from Eqs. (5), (7), and (8), it follows that

$$
\begin{aligned}
& 3 H^{2} \simeq V(\phi), \\
& \dot{\phi} \simeq-\frac{V^{\prime}(\phi)}{3 H(1+Q)}, \\
& \rho_{r}=C_{\gamma} T^{4}=\frac{\Gamma}{4 H} \dot{\phi}^{2},
\end{aligned}
$$

where $T$ is the temperature of the fluid, $C_{\gamma}=\pi^{2} g_{\star} / 30$ is the Stefen-Boltzman constant, and $g_{\star}$ is the number of degrees of freedom of the radiation field. 
With the use of the above equations, it follows that the slow-roll parameters can be expressed as

$\varepsilon_{1}=\frac{1}{2(1+Q)} \frac{V^{\prime 2}(\phi)}{V^{2}(\phi)}, \quad \varepsilon_{2}=\frac{\dot{\varepsilon}_{1}}{H \varepsilon_{1}}$.

It is common to use two other slow-roll parameters, which are stated in terms of the potential as,

$\eta=\frac{1}{(1+Q)} \frac{V^{\prime \prime}}{V}, \quad \beta=\frac{1}{(1+Q)} \frac{V^{\prime} \Gamma^{\prime}}{V \Gamma}$.

The slow-roll parameter $\varepsilon_{2}$ is related to the above slow-roll parameters through the relation

$\varepsilon_{2}=-2 \eta+4 \varepsilon_{1}+\frac{Q}{(1+Q)}\left(\beta-\varepsilon_{1}\right)$.

Due to the appearance of the term $(1+Q)$ in the dominator of the slow-roll parameters, it follows that the smallness of the parameters are guaranteed for a large range of potentials that could satisfy the slow-roll approximations.

Smallness of the slow-roll parameters ensures us that first the Universe had a quasi-exponential accelerated expansionary phase, and also it did stand in this phase for enough number of e-folds to solve the issues of the hot big bang model. The number of e-folds is given by the following relation,

$$
\begin{aligned}
N & =\int_{t_{\star}}^{t_{e}} H d t=\int_{\phi_{\star}}^{\phi_{e}} \frac{H}{\dot{\phi}} d \phi \\
& =-\int_{\phi_{\star}}^{\phi_{e}}(1+Q) \frac{V(\phi)}{V^{\prime}(\phi)} d \phi,
\end{aligned}
$$

where the last equality is obtained by using Eqs. (11) and (10). The subscripts " $e$ " and " $\star$ " respectively indicate the quantity at the end of inflation, and at the horizon crossing time, respectively.

\subsection{Warm inflation with a chameleon-like scalar field}

In the chameleon scalar field model, there is a source term on the right hand side of the conservation Eq. (4), which automatically appears in the equation because of the interaction between the scalar field and the matter sector. Since the chameleon scalar field is taken as the inflaton, it follows that our source term in the conservation equation of the chameleon model plays the same role of the term $\Gamma \dot{\phi}^{2}$ in Eq. (7). Then, we are going to equate these two terms, i.e.

$\Gamma \dot{\phi}^{2}=-\frac{1}{3} \rho_{r s} \frac{\dot{f}(\phi)}{f(\phi)}$,

where the second fluid component of the very early Universe is taken as radiation, with equation of state $p_{r}=\rho_{r} / 3$. On the other hand, the sign of this interaction also shows up in the Friedmann equation, i.e., via the presence of the coupling function $f(\phi)$ in Eq. (1). To get back to the original evolution equation, we can define again an effective radiation energy density and pressure as $\rho_{r s}$ and $p_{r s}$. In this case, the evolution equations are reorganized as

$\dot{\rho}_{r s}+3 H\left(\rho_{r s}+p_{r s}\right)=-p_{r s} \frac{\dot{f}(\phi)}{f(\phi)}=\Gamma \dot{\phi}^{2}$,
$\dot{\rho}_{\phi}+3 H\left(\rho_{\phi}+p_{\phi}\right)=p_{r s} \frac{\dot{f}(\phi)}{f(\phi)}=-\Gamma \dot{\phi}^{2}$.

Assuming a quasi-stable production of the radiation, so that $\dot{\rho}_{r s} \ll H \rho_{r s}$ and $\dot{\rho}_{r s} \ll \Gamma \dot{\phi}^{2}$, which implies that $\dot{\rho}_{r s} \ll$ $p_{r s} \dot{f}(\phi) / f(\phi)$, from Eq. (17) one can calculate the Hubble parameter as

$H=-\frac{1}{12} \frac{\dot{f}(\phi)}{f(\phi)}$.

Using Eq. (10), the potential of the scalar field is derived in terms of $f(\phi)$ and its derivative as

$V(\phi)=\frac{1}{48}\left[\frac{\dot{f}(\phi)}{f(\phi)}\right]^{2}$.

On the other hand, using the result of QFT in curved space, the dissipation coefficient is taken as its best known expression $\Gamma=\Gamma_{0} T^{m} / \phi^{m-1}$. Then, by applying Eqs. (11), (12) and (16), the temperature of the radiation fluid is obtained as

$T^{m-4}=\frac{C_{\gamma}}{\Gamma_{0}}(1+Q) H \frac{f^{\prime}(\phi)}{f(\phi)} \frac{\phi^{m-1}}{V^{\prime}(\phi)}$,

where we have used the mathematical result $\dot{f}(\phi)=f^{\prime}(\phi) \dot{\phi}$.

To go one step further, we assume the ansatz $\dot{f}(\phi) / f(\phi)=$ $\alpha \phi^{n}$. With the use of this assumption in Eqs. (19) and (20), the Hubble parameter and the potential of the scalar field are obtained as

$H=-\frac{\alpha}{12} \phi^{n}, \quad V(\phi)=\frac{\alpha^{2}}{48} \phi^{2 n}$.

Since the Hubble parameter is positive, $\alpha$ should be a negative constant.

On the other hand, with the use of Eqs. (11), (19) and (22) in Eq. (21), one arrives at the following non-linear equation for the temperature of the fluid,

$$
\begin{aligned}
& T^{m}+\frac{\phi^{\frac{m+3 n-3}{2}}}{\xi \Gamma_{0}} T^{\frac{m-4}{2}}-\frac{\alpha}{4 \Gamma_{0}} \phi^{m+n-1}=0, \\
& \xi \equiv\left(-\frac{192 C_{\gamma}}{n^{2} \alpha^{3} \Gamma_{0}}\right)^{1 / 2} .
\end{aligned}
$$

Due to the fact that the third term of the above equation is a power-law function of the scalar field, it follows that the temperature $T(\phi)$ should be a power-law function of the scalar field as well, in order to satisfy the equation. Hence, for the temperature we assume the functional form $T(\phi)=$ $T_{0} \phi^{q}$. By substituting $T$ in Eq. (21), we obtain

$T_{0}^{m} \phi^{m q}+\frac{T_{0}^{\frac{m-4}{2}}}{\xi \Gamma_{0}} \phi^{\frac{m+3 n-3}{2}+\frac{q(m-4)}{2}}-\frac{\alpha}{4 \Gamma_{0}} \phi^{m+n-1}=0$. 
Then, the power of the scalar field of the three terms should be equal, which gives the following relations:

(a) equality of the first and second terms: $q=\frac{m+3 n-3}{m+4}$;

(b) equality of the first and third terms: $q=\frac{m+n-1}{m}$; and

(c) equality of the second and third terms: $q=\frac{m-n+1}{m-4}$.

Hence, if the three conditions are satisfied, the proposed function for the temperature might be a proper solution. However, it can be shown that these conditions actually give one constraint that is extracted as follows:

$$
\begin{aligned}
-\mathrm{a} & =\mathrm{b}: m=\frac{2(n-1)}{n-3} ; \\
-\mathrm{a} & =\mathrm{c}: m=\frac{2(n-1)}{n-3} ; \text { and } \\
-\mathrm{b} & =\mathrm{c}: m=\frac{2(n-1)}{n-3} .
\end{aligned}
$$

Therefore, for the proposed functional form of the temperature, the following constraints must be satisfied:

$m=\frac{2(n-1)}{n-3}, \quad q=\frac{n-1}{2}$,

which show that by determining the power $n$, the power parameters $m$ and $q$ are also determined.

In the warm inflationary scenario, there are both quantum and thermal fluctuations. Thermal fluctuations are dependent on the fluid temperature $T$, and quantum fluctuations depend on the Hubble parameter $H$. A feature of warm inflation is that the fluid temperature is bigger than the Hubble parameter, $T>H$, stating that the thermal fluctuations overcome quantum fluctuations, and become the origin of the Universe's LSS. To ensure that we stay in the warm inflation regime, the condition $T / H>1$

$\frac{T(\phi)}{H(\phi)}=-\frac{12 T_{0}}{\alpha} \phi^{q-n}>1$,

must be satisfied during the cosmological expansion.

The amount of cosmic expansion during inflation is measured through the number of e-folds $N$ defined as

$$
\begin{aligned}
N & =\int_{t_{\star}}^{t_{e}} H d t=\int_{\phi_{\star}}^{\phi_{e}} \frac{H}{\dot{\phi}} d \phi=-\frac{1}{12} \int_{\phi_{\star}}^{\phi_{e}} \frac{f^{\prime}(\phi)}{f(\phi)} d \phi \\
& =-\frac{1}{12} \ln \left[\frac{f\left(\phi_{e}\right)}{f\left(\phi_{\star}\right)}\right] .
\end{aligned}
$$

Once the expression of the scalar field at the end of inflation is obtained from the relation $\varepsilon_{1}\left(\phi_{e}\right)=1$, Eq. (26) is used to derive the scalar field at the time of the horizon crossing in terms of the number of e-folds. Then, all the perturbation parameters could be expressed in terms of the number of e-folds.

To test the validity of a theoretical model, one has to compare its predictions with the observational data. In this regard, we will obtain some important perturbations parameters, such as the amplitude of scalar perturbations, the scalar spectral index and the tensor-to-scalar ratio, and they will be compared with the Planck-2018 data. Following [94,95], the amplitude of scalar perturbations is calculated as

$$
\mathscr{P}_{S}=\left(\frac{H^{2}}{2 \pi \dot{\phi}}\right)^{2}\left(1+2 n_{B E}+\frac{2 \sqrt{3} \pi Q}{\sqrt{3+4 \pi Q}} \frac{T}{H}\right) G(Q),
$$

where $n_{B E}$ is the Bose-Einstein distribution, given by $n_{B E}=$ $\left[\exp \left(H / T_{\delta \phi}\right)-1\right]^{-1}$, and $T_{\delta \phi}$ is the temperature of the inflaton fluctuations [95]. Here $G(Q)$, giving the growth of the fluctuations, is a function of $Q$ and its presence is due to the coupling of the scalar field and radiation [94,95]. The scalar spectral index and its running are obtained from the amplitude of scalar perturbations, and it is defined as

$n_{s}-1=\frac{d \ln \left(\mathscr{P}_{s}\right)}{d \ln (k)}, \quad \alpha_{s}=\frac{d n_{s}}{d \ln (k)}$.

Tensor perturbations, known as gravitational waves, are measured indirectly through the tensor-to-scalar ratio parameter $r=\mathscr{P}_{t} / \mathscr{P}_{s}$. The amplitude of tensor perturbations is given by [94]

$\mathscr{P}_{t}=\frac{2 H^{2}}{\pi^{2}}$.

The tensor spectral index is defined as

$n_{t}=\frac{d \ln \left(\mathscr{P}_{t}\right)}{d \ln (k)}$.

In the relevant literature, the scenario of warm inflation is usually considered in two regimes, known as the strong and the weak dissipative regimes, where the dissipative ratio is respectively $Q \gg 1$ and $Q \ll 1$. In the subsequent subsections, we are going to consider the discussed model in these two regimes.

\subsection{Weak dissipative regime}

In the weak dissipative regime, the dissipative ratio is much smaller than unity, i.e., $Q \ll 1(\Gamma \ll 3 H)$, and thus we have $(1+Q) \simeq 1$. This approximation makes the evolution equation easier to analyse, since the time derivative of the scalar field is expressed as

$\dot{\phi}=\frac{n \alpha}{6} \phi^{n-1}$.

Also, by taking this approximation into account, and following the same process as used previously, the temperature of the fluid is obtained easily as a function of the scalar field:

$T^{m-4}(\phi)=\frac{-12 C_{\gamma}}{n^{2} \alpha \Gamma_{0}} \frac{1}{\phi^{n-m-1}}$. 
In subsection (3.2), an ansatz was introduced for the model. Using this definition and applying Eq. (31), the coupling function $f(\phi)$ is obtained as an exponential function,

$f(\phi)=f_{0} \exp \left(\frac{3}{n} \phi^{2}\right)$,

where $f_{0}$ is a constant of integration.

The slow-roll parameters, introduced in Eqs. (13) and (14), are obtained as

$\varepsilon_{1}(\phi)=\frac{2 n^{2}}{\phi^{2}}, \quad \eta(\phi)=\frac{(2 n-1)}{n} \frac{1}{\phi^{2}}$,

$\beta(\phi)=\frac{(6 m-n m-4)}{n(m-4)} \varepsilon_{1}(\phi)$.

Inflation ends for $\phi_{e}^{2}=2 n^{2}$, and the scalar field at the horizon crossing is extracted from the number of e-folds. Hence from Eq. (26) one arrives at the expression,

$N=\int_{\phi_{\star}}^{\phi_{e}} \frac{H}{\dot{\phi}} d \phi=-\frac{1}{12} \int_{\phi_{\star}}^{\phi_{e}} \frac{f^{\prime}(\phi)}{f(\phi)} d \phi$,

which subsequently gives

$\phi_{\star}^{2}=2 n^{2}\left(1+\frac{2 N}{n}\right)$.

In the weak dissipative regimes, the parameter $G(Q) \simeq 1$ at the time of horizon crossing. Then, from Eq. (27), the amplitude of scalar perturbations in these regimes is found as

$\mathscr{P}_{s}=2\left(\frac{H^{2}}{2 \pi \dot{\phi}}\right)^{2} \frac{T}{H}$.

From Eq. (28) the scalar spectral index and its running in the weak regimes are obtained as

$n_{s}-1=-\frac{17}{8} \varepsilon_{1}+\frac{3}{2} \eta-\frac{1}{4} \beta$,

where $\eta$ is defined through the slow-roll parameters $\varepsilon_{2}$ as $\eta=V^{\prime \prime} /(1+Q) V$, so that $\varepsilon_{2}=\eta-\varepsilon_{1}$, and the slowroll parameter $\beta$ is given as $\beta=V^{\prime} \Gamma^{\prime} /(1+Q) V \Gamma$. The tensor-to-scalar ratio in this case is also obtained as

$r=8 \varepsilon_{1} \frac{H}{T}$.

To compare the results of the model with observational data, the above perturbation parameters are computed at the horizon crossing. Using Eq. (36), the slow-roll parameters at horizon crossing are obtained as follows:

$$
\begin{aligned}
\varepsilon_{1}^{\star} & =\left(1+\frac{2 N}{n}\right)^{-1}, \\
\eta^{\star} & =\frac{2 n-1}{n} \varepsilon_{1}^{\star}, \\
\beta^{\star} & =-\frac{n m-6 m+4}{n(m-4)} \varepsilon_{1}^{\star} .
\end{aligned}
$$

Substituting the above slow-roll parameters in Eq. (38), the scalar spectral index is given as a function of $n, m$ and efolding number $N$ as

$$
\begin{aligned}
n_{s}(n, m, N)= & 1-\left(\frac{17}{8}-\frac{3(2 n-1)}{2 n}-\frac{n m-6 m+4}{4 n(m-4)}\right) \\
& \times\left(1+\frac{2 N}{n}\right)^{-1} .
\end{aligned}
$$

From Eq. (37), and by using Eq. (36), the model constant parameter $\Gamma_{0}$ is found in terms of $n, m$ and $N$ as

$$
\begin{aligned}
\Gamma_{0}(n, m, N)= & -\frac{12 C_{\gamma}}{\alpha n^{2}}\left(-\frac{\alpha}{96 \pi^{2} n^{2} \mathscr{P}_{s}}\right)^{m-4} \\
& \times\left[2 n^{2}\left(1+\frac{2 N}{n}\right)\right]^{\frac{n m-5 n+3 m-7}{2}},
\end{aligned}
$$

where $\mathscr{P}_{S}$ is the amplitude of the scalar perturbations at horizon crossing. According to the latest observational data $\mathscr{P}_{s}=2.17 \times 10^{-9}$. The tensor-to-scalar ratio for the same time is found from Eq. (39) as

$$
\begin{aligned}
r^{\star}(n, m, N)= & -\frac{4 \alpha n^{2}}{3}\left(-\frac{\alpha n^{2}}{12 C_{\gamma}}\right)^{\frac{1}{m-4}} \Gamma_{0}^{\frac{1}{m-4}}(n, m, N) \\
& \times\left[2 n^{2}\left(1+\frac{2 N}{n}\right)\right]^{\frac{n m-3(n+m)+7}{2(m-4)}} .
\end{aligned}
$$

Using the $r-n_{s}$ diagram of Planck-2018, one could plot a $n-m$ diagram as shown in Fig. 1, where the dark blue color indicates an area of $(n, m)$ where the results for $n_{s}$ and $r$ stand in $68 \% \mathrm{CL}$. The light blue color indicates an area of $(n, m)$ in which the point $\left(r, n_{s}\right)$ of the model stands in $95 \%$ CL.

Inserting the scalar field at horizon crossing in Eq. (20), the potential of the scalar field could be obtained in terms of

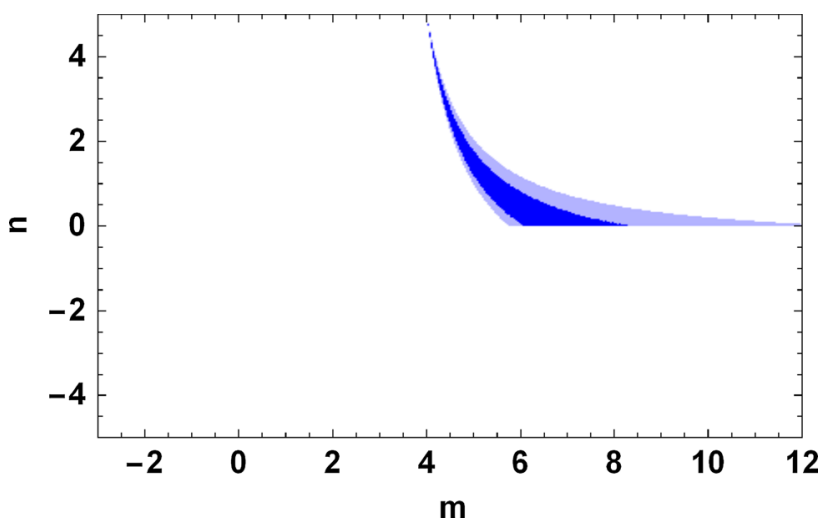

Fig. 1 Numerical values of the $(n, m)$ parameters of the chameleon field driven warm inflation model in the weak dissipative regime for which the point $\left(r-n_{s}\right)$ is located in the observational region. The dark blue color shows the values of $(n, m)$ in the $68 \%$ CL range of the Planck-2018 data, while the light blue color shows the parameter values in the range $95 \% \mathrm{CL}$ 
$N$ for specific values of $(n, m)$ that have been taken from Fig. 2. The results in the Figure indicate that the inflation did begin at an energy scale about $10^{15-16} \mathrm{GeV}$. At the onset of inflation, the scalar field stands on the top, and by passing time it rolls down slowly until the end of inflation.

Inserting the scalar field at horizon crossing in Eq. (33), the coupling function could be depicted versus $N$. Getting some point of $(n, m)$ from Fig. 1, the behavior of the coupling function $f(\phi)$ in the WDR is illustrated in Fig. 3. The curves show the behavior of $\ln (f(N))$ versus $\ln (N)$ during the inflationary era; however, the scales on the axes show the actual ranges of $f(N)$ and $N$. The coupling function $f(N)$ has an exponential behavior, and it grows rapidly by enhancement of $N$.

An important feature of the warm inflationary scenario is that the thermal fluctuations overcome the quantum fluctuations, since the fluid temperature is bigger than the Hubble parameter. To have a healthy warm inflation, this condition should be satisfied during the cosmological evolution. Figure 4 describes the behavior of the ratio of the temperature to the Hubble parameter during this era. From the Figure one can see that the condition is satisfied during this phase of cosmological expansion.

\subsection{Strong dissipative regime}

In this regime, the dissipative ratio is much larger than unity, so that $(1+Q) \simeq Q$. In this case, the analysis of the model becomes more complicated, and the cosmological dynamics is different from the weak dissipative regime. In this approximation the time derivative of the scalar field is given by

$\dot{\phi}=-\frac{V^{\prime}(\phi)}{\Gamma}$.

Using Eq. (16), and by considering the definition of $\Gamma$, and Eq. (46), the temperature of the radiation fluid is obtained as

$T^{m+4}(\phi)=-\frac{n^{2} \alpha^{3}}{192 \Gamma_{0} C_{\gamma}} \phi^{3 n+m-3}=T_{0}^{m+4} \phi^{3 n+m-3}$.

Then, with the help of this relation, the time derivative of the scalar field can be found in terms of the scalar field, so that

$\dot{\phi}=-\frac{n \alpha^{2}}{24 \Gamma_{0} T_{0}^{m}} \phi^{\frac{8 n-n m+5 m-8}{m+4}}$.

The dissipative ratio $Q$ is given by

$Q(\phi)=\frac{\Gamma}{3 H}=-\frac{4 \Gamma_{0} T_{0}^{m}}{\alpha} \phi^{\frac{2(n m-2 n-3 m+2)}{m+4}}$.

From Eqs. (13) and (14), after substituting Eq. (49), we find out the slow-roll parameters as

$\varepsilon_{1}(\phi)=-\frac{\alpha n^{2}}{2 \Gamma_{0} T_{0}^{m}} \phi^{\frac{-2(n m-2 n-2 m+6)}{m+4}}$,

$$
\begin{aligned}
\eta(\phi) & =\frac{(2 n-1)}{n} \varepsilon_{1}(\phi), \\
\beta(\phi) & =\frac{m(3 n-6)+4}{n(m+4)} \varepsilon_{1}(\phi) .
\end{aligned}
$$

Then, the scalar field at the end of inflation is obtained as

$$
\phi_{e}^{\frac{2(n m-2 n-2 m+6)}{m+4}}=-\frac{\alpha n^{2}}{2 \Gamma_{0} T_{0}^{m}} \text {. }
$$

After obtaining $\dot{\phi}(\phi)$ and $Q(\phi)$, the coupling function $f(\phi)$ can also be determined through the definition introduced previously. It turns out that $f(\phi)$ is again an exponential function of the scalar field, and it is given by

$$
\begin{aligned}
f(\phi)= & f_{0} \exp \\
& \times\left(-\frac{12 \Gamma_{0} T_{0}^{m}}{n \alpha} \frac{m+4}{n m-2 n-2 m+6} \phi^{\frac{2(n m-2 n-2 m+6)}{m+4}}\right),
\end{aligned}
$$

where $f_{0}$ is an arbitrary constant of integration. Using this result in the relation of $N$, after integration, the scalar field at the time of horizon crossing is obtained in terms of $N$ as

$$
\begin{aligned}
\phi_{\star}^{\frac{2(n m-2 n-2 m+6)}{m+4}}= & -\frac{\alpha n^{2}}{2 \Gamma_{0} T_{0}^{m}} \\
& \times\left(1+\frac{2(n m-2 n-2 m+6)}{n(m+4)} N\right) .
\end{aligned}
$$

In the strong dissipative regime, the parameter $G(Q)$, depending on the different values of the parameter $m$, can be approximated as $[94,95]$ :

$$
\begin{aligned}
m=1 & \longrightarrow G(Q) \simeq 1+0.127 Q^{4.330}+4.981 Q^{1.946}, \\
m=3 \longrightarrow G(Q) & \simeq 1+0.0185 Q^{2.315}+0.335 Q^{1.364}, \\
m=-1 \longrightarrow G(Q) & \simeq \frac{1+0.4 Q^{0.77}}{\left(1+0.15 Q^{1.09}\right)^{2}} .
\end{aligned}
$$

Therefore, in a more convenient way, we can write down the function $G(Q)=a_{m} Q^{b_{m}}$, where

$$
\begin{gathered}
m=1 \longrightarrow a_{m}=0.127, \quad b_{m}=4.330, \\
m=3 \longrightarrow a_{m}=0.0185, \quad b_{m}=2.315, \\
m=-1 \longrightarrow a_{m}=17.78, \quad b_{m}=-1.41 .
\end{gathered}
$$

For the case $m=-1$, the power of the term $Q$ is negative stating that the term $G(Q)$ tends to zero which in turn lead the amplitude of the scalar perturbation to zero. So, this case is not considered here.

Hence the amplitude of the scalar perturbations is obtained as

$$
\mathscr{P}_{s}=\left(\frac{H^{2}}{2 \pi \dot{\phi}}\right)^{2} \sqrt{3 \pi Q} \frac{T}{H} \times a_{m} Q^{b_{m}} .
$$

By taking the time derivative of this equation according to Eq. (28) leads to the scalar spectral index and its running, given by 
Fig. 2 The behavior of the scalar field potential $V(\phi)$ versus $\phi$ of the chameleon field driven warm inflation model in the weak dissipative regime for different values of $n$ during inflation. The figure shows that for $n=2$, the energy scale of inflation is about $10^{15} \mathrm{GeV}$. Once inflation is approaching to its end, the potential decreases in time

Fig. 3 The behavior of the coupling function $f(N)$ versus the number of e-fold for different values of $n$ (in a logarithmic scale). The function has an exponential behavior and when approaching to the end of inflation it rapidly decreases
Fig. 4 The ratio of the temperature to the Hubble parameter during the inflationary period of the chameleon field driven warm inflation model in the weak dissipative regime versus the number of e-folds for different values of $(n, m)$. As one can see from the plots during inflation the temperature is larger than the Hubble parameter, and the condition $T>H$ is satisfied properly

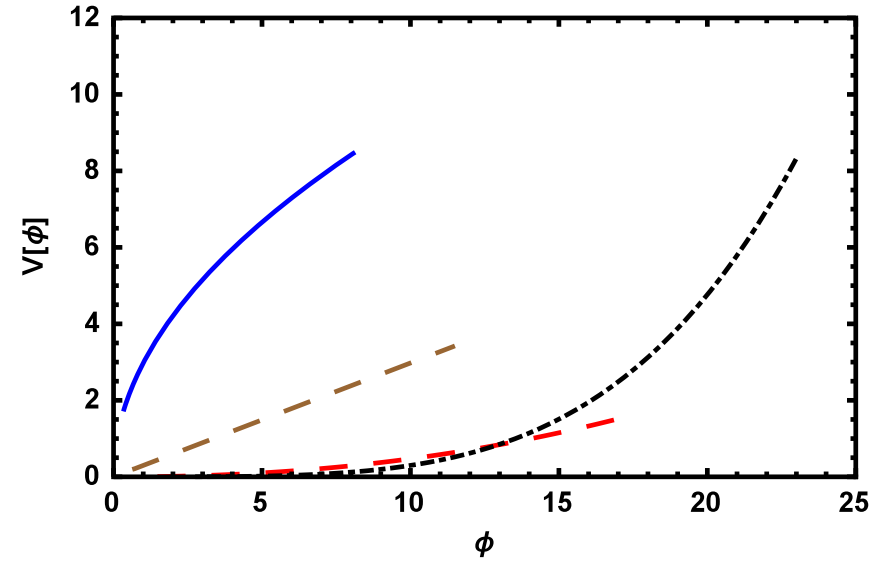

$$
\begin{aligned}
& -\quad n=0.50 ; \mathrm{V} \times 10^{-16} \\
& ---n=1.10 ; \mathrm{V} \times 10^{-14} \\
& -n=2.00 ; \mathrm{V} \times 10^{-12}
\end{aligned}
$$

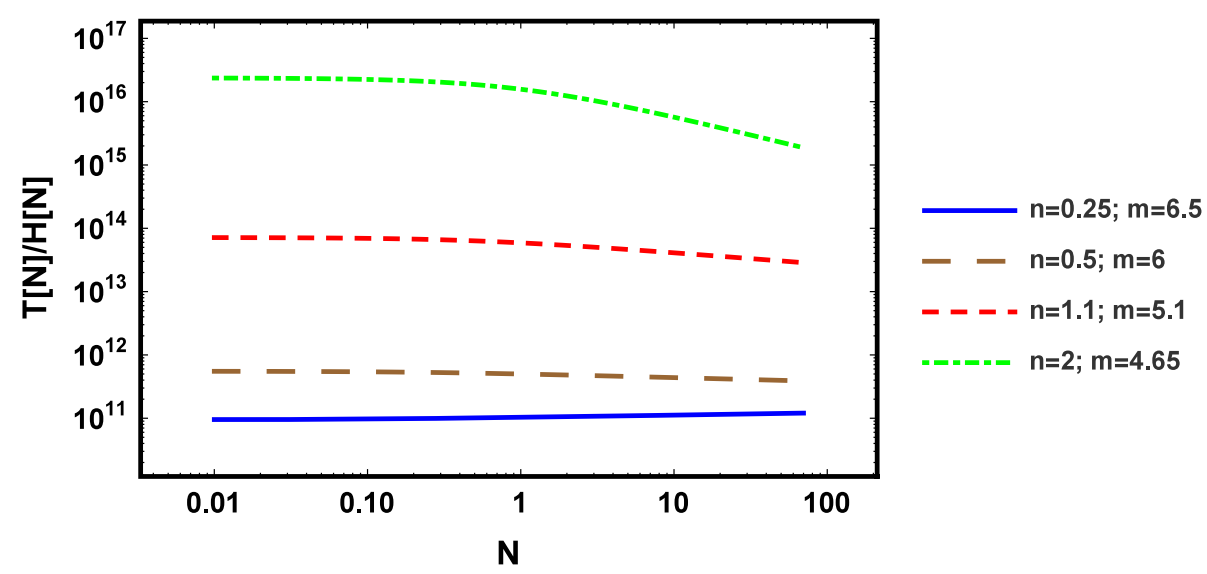

$n_{s}-1=-2 q_{1} \varepsilon_{1}-q_{2} \beta+\frac{3}{2} \eta$,

where $q_{1}=\frac{9}{8}-\frac{b_{m}}{2}$ and $q_{2}=b_{m}+\frac{7}{4}$. We notice that the function $G(Q)$ for $m=3$ has an acceptable behaviour for the value $m=-1$, or even for $m=1$. The amplitude of tensor perturbations in strong dissipative regimes is given by $[94,95]$

$\mathscr{P}_{t}=\frac{2 H^{2}}{\pi^{2}}=\frac{\alpha^{2}}{72 \pi^{2}} \phi^{2 n}$.

Then, the tensor-to-scalar ratio is obtained as follows:

$r=\frac{24(\sqrt{3})^{b_{m}}}{\sqrt{3 \pi} a_{m}}\left(\frac{4 C_{\gamma}}{9}\right)^{\frac{1}{4}} \frac{V^{\prime 3 / 2}}{V^{\left(q_{1}-1\right)} \Gamma^{q_{2}}}$.

Using Eq. (54), the above perturbation parameters can be obtained at the horizon crossing time. The slow-roll parameters in the SDR at this cosmological instance are given by

$\varepsilon_{1}^{\star}=\left(1+\frac{2(n m-2 n-2 m+6) N}{n(m+4)}\right)^{-1}$, 


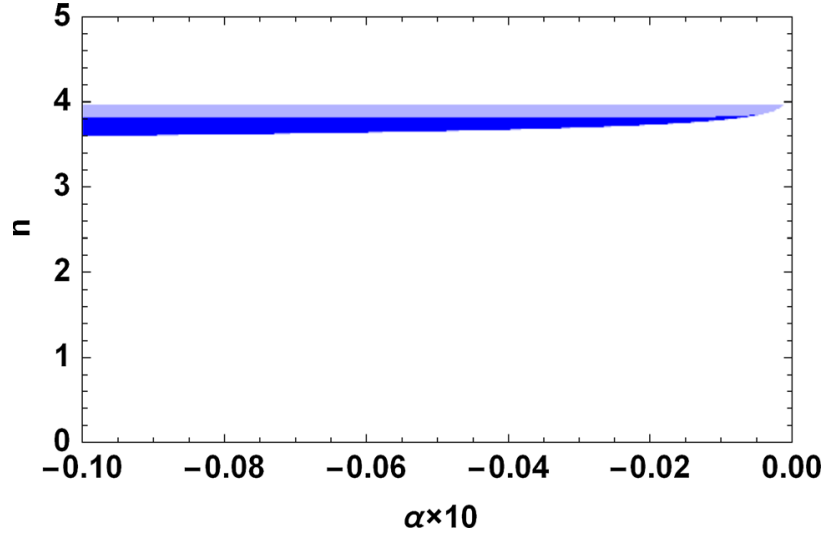

Fig. 5 The ranges of the parameters $(n, \alpha)$ of the chameleon field driven warm inflation model in the strong dissipative regime that are consistent with $n_{s}$ and $r$ located in the observational area. The light blue color indicates the areas with 95\% CL, while the dark blue color indicates the areas with $68 \% \mathrm{CL}$

$\eta^{\star}=\frac{2 n-1}{n} \varepsilon_{1}^{\star}$

$\beta^{\star}=\frac{3 n m-6 m+4}{n(m+4)} \varepsilon_{1}^{\star}$.

Hence we obtain the following scalar spectral index,

$$
\begin{aligned}
& n_{s}(n, \alpha, N) \\
& \quad=1-\left(2 q_{1}+\frac{3 n m-6 m+4}{n(m+4)} q_{2}-\frac{3(2 n-1)}{2 n}\right) \varepsilon_{1}^{\star} .
\end{aligned}
$$

We notice that this is a function of $n, m$ and $N$. The constant parameter $\Gamma_{0}$ can be determined by using the observational data for the amplitude of the scalar perturbations as

$$
\begin{aligned}
& \Gamma_{0}(n, \alpha, N) \\
& =\frac{B}{\mathscr{P}_{s}^{\star}}\left(-\frac{n^{2} \alpha}{2}\left[-\frac{192 C_{\gamma}}{n^{2} \alpha^{3}}\right]^{\frac{m}{m+4}} \frac{1}{\varepsilon_{1}^{\star}}\right)^{\frac{(m+4) \sigma}{2 S(n m-2 n-2 m+6)}},
\end{aligned}
$$

where

$$
\begin{aligned}
B(n, \alpha, N)= & \frac{1}{(2 n)^{3 / 2}}\left(\frac{9}{4 C_{\gamma}}\right)^{\frac{1}{4}} \frac{\sqrt{3 \pi} a_{m}}{36(\sqrt{3})^{b_{m}} \pi^{2}}\left(\frac{\alpha^{2}}{48}\right)^{q_{1}-\frac{3}{2}} \\
& \times\left[-\frac{192 C_{\gamma}}{n^{2} \alpha^{3}}\right]^{\frac{-m q_{2}}{m+4}}, \\
S(n, \alpha, N)= & \frac{4 q_{2}}{m+4}-\frac{4 \sigma}{(n m-2 n-2 m+6)},
\end{aligned}
$$

and

$\sigma(n, \alpha, N)=-\frac{3(2 n-1)}{2}+2 n q_{1}+\frac{(3 n m-6 m+4)}{m+4} q_{2}$,

respectively. Using the above relations, the tensor-to-scalar ratio at the horizon crossing is obtained as

$$
\begin{aligned}
& r^{\star}(n, \alpha, N)=\frac{\alpha^{2}}{72 \pi^{2}} \frac{B^{-1}}{\mathscr{P}_{s}^{\star}} \\
& \quad \times\left(-\frac{n^{2} \alpha}{2}\left[\frac{192 C_{\gamma}}{-n^{2} \alpha^{3}}\right]^{\frac{m}{m+4}} \frac{1}{\varepsilon_{1}^{\star}}\right)^{\frac{(m+4)(2 n-\sigma)}{2(n m-2 n-2 m+6)}} \Gamma_{0}^{\tilde{S}},
\end{aligned}
$$

where we have defined

$\tilde{S}(n, \alpha, N)=-\frac{4 q_{2}}{m+4}-\frac{2(2 n-\sigma)}{(n m-2 n-2 m+6)}$.

Similarly as in the previous section, we use the $r-n_{s}$ diagram of Planck-2018 to find out the proper region of $(n, \alpha)$, which leads for the model prediction to be agreement with observational data. Figure 5 shows this area, depicted with dark and light blue colors, respectively, displaying the values of $(n, \alpha)$ compatible with $68 \%$ and $95 \%$ CL, respectively. With the use of the value of the scalar field at horizon crossing, i.e. substituting Eq. (54) in Eq. (20), and by choosing the proper values of $(n, \alpha)$ from Fig. 5 , the scalar field potential is expressed in terms of the number of e-folds. Figure 6 depicts the behavior of the scalar field potential during inflation, indicating that the inflation energy scale could be about $10^{15-16} \mathrm{GeV}$.
Fig. 6 The behavior of the scalar field potential $V(\phi)$ versus the inflation scalar field in the chameleon field driven warm inflation model in the strong dissipative regime for different values of $(n, \alpha)$, obtained from Fig. 5. The figure shows that the inflation could start from energy scales of the order of $10^{15} \mathrm{GeV}$, with the scalar field potential decreasing during the cosmological evolution

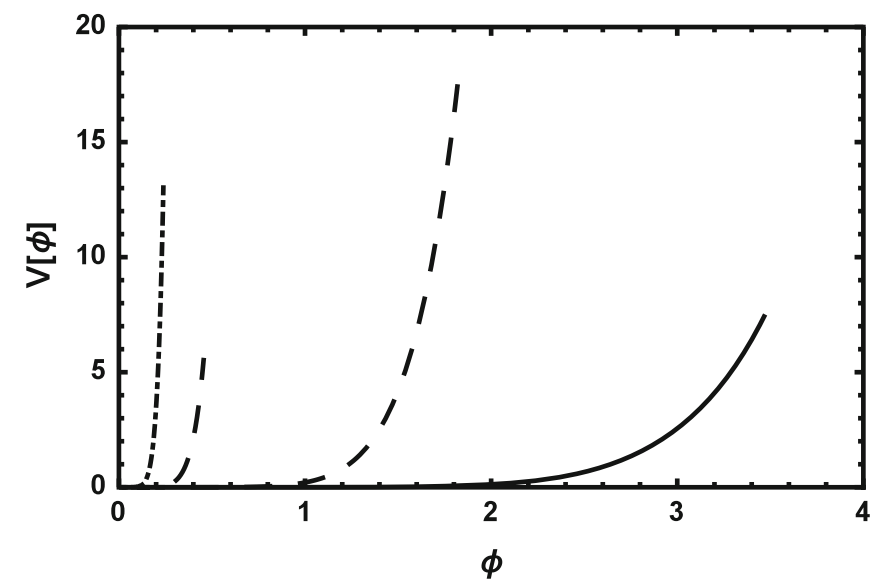


Fig. 7 The behavior of coupling function $f(N)$ versus the number of e-folds in the chameleon field driven warm inflation model in the strong dissipative regime for different values of $(n, \alpha)$, which are selected from Fig. 5. The function $f(N)$ has an exponential behavior, and during inflation it decreases

Fig. 8 The ratio of the temperature to the Hubble parameter during the inflationary evolution of the chameleon field driven warm inflation model in the strong dissipative regime versus the number of e-folds for different values of $(n, \alpha)$. During inflation the temperature is bigger than the Hubble parameter, and their ratio increases when approaching the end of inflation
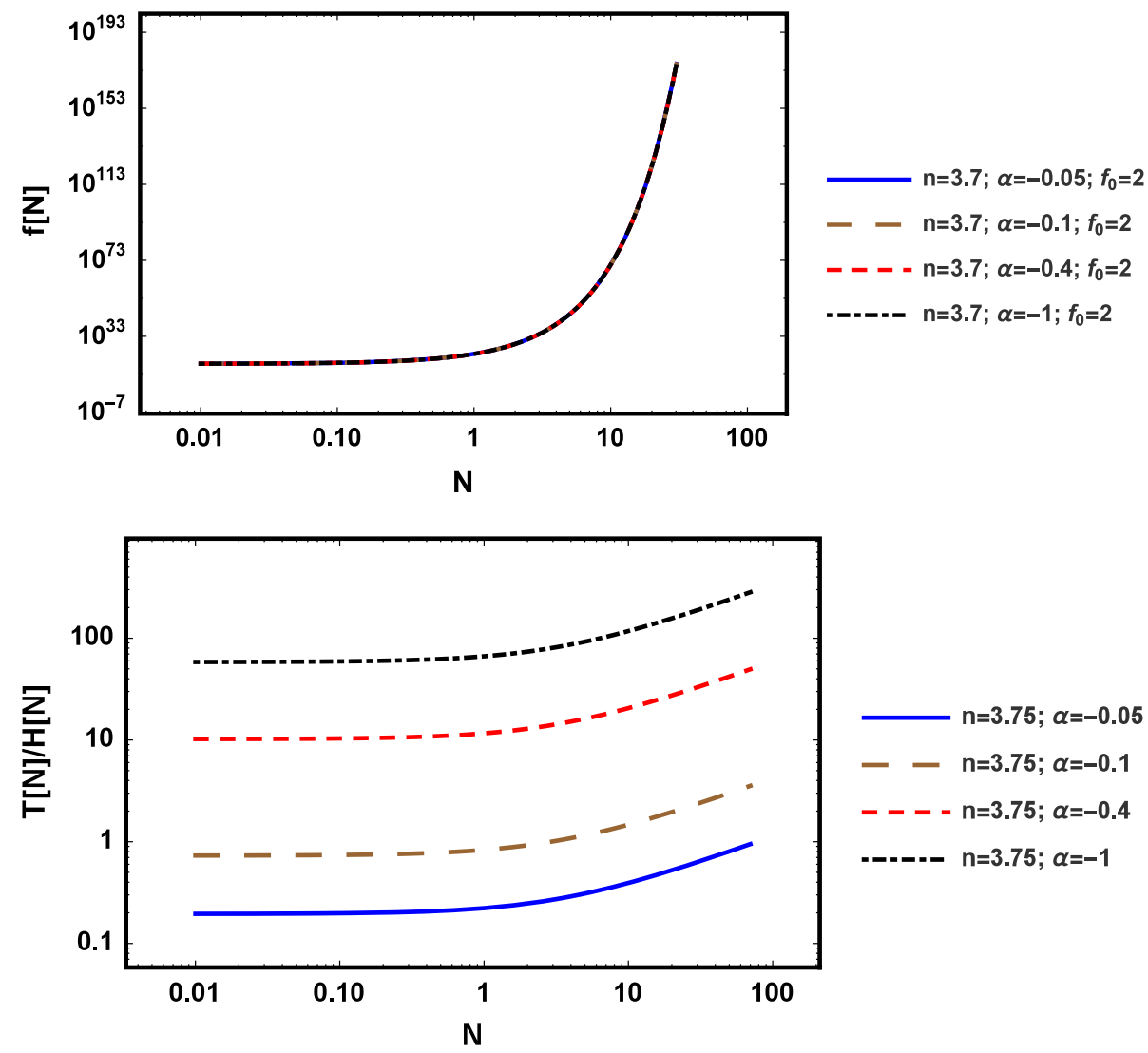

By using the value of the scalar field at horizon crossing, i.e. Eq. (54) in Eq. (53), the coupling function $f(\phi)$ can again be expressed in terms of the number of e-folds. Figure 7 portrays the behavior of the coupling function during inflation for different values of $(n, \alpha)$ that have been selected from Fig. 5. The curves depict the behavior of $\ln [f(N)]$ versus $\ln (N)$ during the inflationary times, however, the scales show the true values of $f(N)$ and $N$. The coupling function $f(N)$ has an exponential behavior and it grows rapidly by the enhancement of $N$

As the final step, the condition $T / H>1$ is considered by plotting the ratio of the temperature to the Hubble parameter versus the number of e-folds during inflation, as shown in Fig. 8. From the Figure it immediately follows that the condition is satisfied during the cosmological evolution.

\section{Conclusion}

In the present work, we have studied the behavior of chameleon-like scalar fields, non-minimally interacting with matter, as potential candidates for driving warm inflationary evolution. Since the scalar field interacts with the matter sector, in these models one has a generalized energy conservation law, where there is a source for the scalar field, depending on the scalar field - matter coupling function, and on the matter Lagrangian. Besides, due to this interaction, the mass of the scalar field strongly depends on the environment energy density, so that on cosmological length scales, the scalar field mass is small, while on short scales, like, for example, around the Earth, the scalar field mass grows. This interesting feature of the chameleon scalar field model may be of major interest for cosmology.

The scenario of warm inflation is a different story compared to the cold one. Although this model has almost the same basic assumptions, here the scalar field interacts with the matter sector during inflation, and energy transfer from the scalar field to the matter part does occur during the accelerated expansion phase. Due to this feature, the chameleon scalar field model seems to be a suitable model for describing warm inflation, since it naturally predicts such an interaction. By using quantum field theory and particle physics, it can be shown that the dissipation coefficient, in general, depends on both scalar field and temperature. Applying this result, and imposing an appropriate ansatz, the Hubble parameter, the scalar field potential, the coupling function, and the temperature can be obtained in terms of the scalar field. Then, the chameleon field driven warm inflationary scenario was considered in detail in two approximations, corresponding to the weak and strong dissipative regimes, respectively. The main 
perturbation parameters, such as the amplitude of scalar perturbations, the scalar spectral index, and tensor-to-scalar ratio were derived for each regime. After computing the scalar spectral index and tensor-to-scalar ratio, and by using the $r-n_{s}$ diagram of Planck-2018, we plotted the area of the model parameters $(n, m)$ and $(n, \alpha)$ that places the model exactly in the $68 \%$ and $95 \%$ CL areas, which are depicted in Figs. 1 and 5, respectively, corresponding to the weak and strong dissipative regimes.

Using the results on the model parameters, the scalar field potentials were obtained for both regimes. Our results show that the inflation energy scale could be around $10^{15} \mathrm{GeV}$, and the scalar field stands on the top of its potential, and rolls down slowly during the cosmological evolution, when and approaching to the end of inflation. Also, the results of the investigation of the coupling function $f(\phi)$ shows that it has an exponential behavior during inflation in both regimes.

Another difference between warm inflation and cold inflation is about the type of perturbations. In cold inflation, the quantum perturbations are produced during inflation, and these perturbation are the seeds for LSS of the Universe. On the other hand, in warm inflation, there are both quantum and thermal perturbations, and it is the thermal perturbations that dominate, and are the basic seeds for the formation of the LSS of the Universe. This domination is expressed by the condition $T>H$, otherwise, the quantum fluctuations are overwhelmed and we do not have warm inflation anymore. So, this condition as an important feature of warm inflation was studied as well. Our investigations show that during inflation the temperature is larger than the Hubble parameter, and this result has been illustrated in Figs. 4 and 8, respectively.

The initial conditions of the inflation, as well as the matter content at the moment of the birth of the Universe are not fully known. Similarly, there are many theoretical and observational uncertainties in the physics of the decay and interactions of the scalar fields. Probably a full understanding of these processes may need physics beyond the Standard Model of particle physics to account for the radiation and other forms of matter creation process. And, to constrain the theoretical models, and find out what truly happened in the very early Universe. certainly more accurate observational data are needed. In the present investigation we have proposed some basic tools that may open the possibility of an in-depth comparison of the predictions of the theoretical models of inflation with the cosmological data.

Acknowledgements HS thanks A. Starobinsky for very constructive discussions about inflation during Helmholtz International Summer School 2019 in Russia. He grateful G. Ellis, A. Weltman, and UCT for arranging his short visit, and for enlightening discussions about cosmological fluctuations and perturbations for both large and local scales. He also thanks IPM, specially H. Firouzjahi for constructive discussions about inflation and perturbations. His special thanks go to his wife E. Avirdi for her patience during our stay in South Africa. AM would like to thank the Ministry of Science, Research and Technology of Iran for financial support during his visit to the University of Bologna. He also thanks Prof. R. Casadio and the department of Physics and Astronomy of the University of Bologna for their kind hospitality. Christian Corda has been supported financially by the Research Institute for Astronomy and Astrophysics of Maragha (RIAAM). AA acknowledges that this work is based on the research supported in part by the National Research Foundation (NRF) of South Africa (Grant numbers 109257 and 112131).

Data Availability Statement This manuscript has no associated data or the data will not be deposited. [Authors' comment: The datasets generated during and/or analysed during the current study are available from the corresponding author on reasonable request.]

Open Access This article is licensed under a Creative Commons Attribution 4.0 International License, which permits use, sharing, adaptation, distribution and reproduction in any medium or format, as long as you give appropriate credit to the original author(s) and the source, provide a link to the Creative Commons licence, and indicate if changes were made. The images or other third party material in this article are included in the article's Creative Commons licence, unless indicated otherwise in a credit line to the material. If material is not included in the article's Creative Commons licence and your intended use is not permitted by statutory regulation or exceeds the permitted use, you will need to obtain permission directly from the copyright holder. To view a copy of this licence, visit http://creativecomm ons.org/licenses/by/4.0/.

Funded by SCOAP 3 .

\section{References}

1. A.H. Guth, The inflationary universe: a possible solution to the horizon and flatness problems. Phys. Rev. D 23, 347-356 (1981)

2. A.D. Linde, A new inflationary universe scenario: a possible solution of the horizon, flatness, homogeneity, isotropy and primordial monopole problems. Phys. Lett. B 108, 389-393 (1982)

3. A. Albrecht, P.J. Steinhardt, Cosmology for grand unified theories with radiatively induced symmetry breaking. Phys. Rev. Lett. 48, 1220-1223 (1982)

4. A.D. Linde, Chaotic inflation. Phys. Lett. B 129, 177-181 (1983)

5. A.D. Linde, Inflationary cosmology. Lect. Notes Phys. 738, 1-54 (2008). arXiv:0705.0164

6. D. Kazanas, Dynamics of the universe and spontaneous symmetry breaking. Astrophys. J. 241, L59 (1980)

7. J. Martin, Inflation and precision cosmology. Braz. J. Phys. 34, 1307-1321 (2004). arXiv:astro-ph/0312492

8. J. Martin, Inflationary cosmological perturbations of quantummechanical origin. Lect. Notes Phys. 669, 199-244 (2005). arXiv:hep-th/0406011

9. J. Martin, Inflationary perturbations: the cosmological Schwinger effect. Lect. Notes Phys. 738, 193-241 (2008). arXiv:0704.3540

10. A.A. Starobinsky, Relict gravitation radiation spectrum and initial state of the universe (In Russian). JETP Lett. 30, 682-685 (1979)

11. V.F. Mukhanov, G. Chibisov, Quantum fluctuation and nonsingular universe (In Russian). JETP Lett. 33, 532-535 (1981)

12. S. Hawking, The development of irregularities in a single bubble inflationary universe. Phys. Lett. B 115, 295 (1982). (Revised version)

13. A.A. Starobinsky, Dynamics of phase transition in the new inflationary universe scenario and generation of perturbations. Phys. Lett. B 117, 175-178 (1982) 
14. A.H. Guth, S.Y. Pi, Fluctuations in the new inflationary universe. Phys. Rev. Lett. 49, 1110-1113 (1982)

15. J.M. Bardeen, P.J. Steinhardt, M.S. Turner, Spontaneous creation of almost scale-free density perturbations in an inflationary universe. Phys. Rev. D 28, 679 (1983)

16. J. Martin, C. Ringeval, V. Vennin, Encyclopædia inflationaris. Phys. Dark Univ. 5-6, 75 (2014). https://doi.org/10.1016/j.dark. 2014.01.003. arXiv:1303.3787 [astro-ph.CO]

17. E.D. Stewart, D.H. Lyth, A more accurate analytic calculation of the spectrum of cosmological perturbations produced during inflation. Phys. Lett. B 302, 171-175 (1993). arXiv:gr-qc/9302019

18. V.F. Mukhanov, H.A. Feldman, R.H. Brandenberger, Theory of cosmological perturbations. Part 1. Classical perturbations. Part 2. Quantum theory of perturbations. Part 3. Extensions. Phys. Rep. 215, 203-333 (1992)

19. A.R. Liddle, D.H. Lyth, Cosmological Inflation and Large Scale Structure (Cambridge University Press, Cambridge, 2000), p. 400

20. A.R. Liddle, P. Parsons, J.D. Barrow, Formalizing the slow roll approximation in inflation. Phys. Rev. D 50, 7222-7232 (1994). arXiv:astro-ph/9408015

21. C. Bennett, D. Larson, J. Weiland, N. Jarosik, G. Hinshaw, et al., Nine-year Wilkinson microwave anisotropy probe (WMAP) observations: final maps and results. arXiv: 1212.5225

22. G. Hinshaw, D. Larson, E. Komatsu, D. Spergel, C. Bennett, et al., Nine-year Wilkinson microwave anisotropy probe (WMAP) observations: cosmological parameter results. arXiv:1212.5226

23. L.-M. Wang, M. Kamionkowski, The cosmic microwave background bispectrum and inflation. Phys. Rev. D 61, 063504 (2000). arXiv:astro-ph/9907431

24. A. Gangui, F. Lucchin, S. Matarrese, S. Mollerach, The three point correlation function of the cosmic microwave background in inflationary models. Astrophys. J. 430, 447-457 (1994). arXiv:astro-ph/9312033

25. A. Gangui, NonGaussian effects in the cosmic microwave background from inflation. Phys. Rev. D 50, 3684-3691 (1994). arXiv:astro-ph/9406014

26. C. Kiefer, D. Polarski, A.A. Starobinsky, Quantum to classical transition for fluctuations in the early universe. Int. J. Mod. Phys. D 7, 455-462 (1998). arXiv:gr-qc/9802003

27. C. Kiefer, D. Polarski, Why do cosmological perturbations look classical to us? Adv. Sci. Lett. 2, 164-173 (2009). arXiv:0810.0087

28. D. Sudarsky, Shortcomings in the understanding of why cosmological perturbations look classical. Int. J. Mod. Phys. D 20, 509552 (2011). arXiv:0906.0315

29. J. Martin, V. Vennin, P. Peter, Cosmological inflation and the quantum measurement problem. Phys. Rev. D 86, 103524 (2012). arXiv: 1207.2086

30. J. Martin, The quantum state of inflationary perturbations. J. Phys. Conf. Ser. 405, 012004 (2012). arXiv: 1209.3092

31. S. Alexander, R.H. Brandenberger, D. Easson, Brane gases in the early universe. Phys. Rev. D 62, 103509 (2000). arXiv:hep-th/0005212

32. P.J. Steinhardt, N. Turok, Cosmic evolution in a cyclic universe. Phys. Rev. D 65, 126003 (2002). arXiv:hep-th/0111098

33. J. Khoury, B.A. Ovrut, N. Seiberg, P.J. Steinhardt, N. Turok, From big crunch to big bang. Phys. Rev. D 65, 086007 (2002). arXiv:hep-th/0108187

34. J. Khoury, B.A. Ovrut, P.J. Steinhardt, N. Turok, The Ekpyrotic universe: colliding branes and the origin of the hot big bang. Phys. Rev. D 64, 123522 (2001). arXiv:hep-th/0103239

35. J. Martin, P. Peter, N. Pinto Neto, D.J. Schwarz, Passing through the bounce in the ekpyrotic models. Phys. Rev. D 65, 123513 (2002). arXiv:hep-th/0112128

36. P. Steinhardt, N. Turok, A cyclic model of the universe. Science 296, 1436-1439 (2002)
37. F. Finelli, R. Brandenberger, On the generation of a scale invariant spectrum of adiabatic fluctuations in cosmological models with a contracting phase. Phys. Rev. D 65, 103522 (2002). arXiv:hep-th/0112249

38. R. Brandenberger, D.A. Easson, D. Kimberly, Loitering phase in brane gas cosmology. Nucl. Phys. B 623, 421-436 (2002). arXiv:hep-th/0109165

39. R. Kallosh, L. Kofman, A.D. Linde, Pyrotechnic universe. Phys. Rev. D 64, 123523 (2001). arXiv:hep-th/0104073

40. J. Martin, P. Peter, N. Pinto-Neto, D.J. Schwarz, Comment on 'Density perturbations in the ekpyrotic scenario'. Phys. Rev. D 67, 028301 (2003). arXiv:hep-th/0204222

41. P. Peter, N. Pinto-Neto, Primordial perturbations in a non singular bouncing universe model. Phys. Rev. D 66, 063509 (2002). arXiv:hep-th/0203013

42. S. Tsujikawa, R. Brandenberger, F. Finelli, On the construction of nonsingular pre-big bang and ekpyrotic cosmologies and the resulting density perturbations. Phys. Rev. D 66, 083513 (2002). arXiv:hep-th/0207228

43. L. Kofman, A.D. Linde, V.F. Mukhanov, Inflationary theory and alternative cosmology. JHEP 0210, 057 (2002). arXiv:hep-th/0206088

44. J. Khoury, P.J. Steinhardt, N. Turok, Designing cyclic universe models. Phys. Rev. Lett. 92, 031302 (2004). arXiv:hep-th/0307132

45. J. Martin, P. Peter, On the causality argument in bouncing cosmologies. Phys. Rev. Lett. 92, 061301 (2004). arXiv:astro-ph/0312488

46. J. Martin, P. Peter, Parametric amplification of metric fluctuations through a bouncing phase. Phys. Rev. D 68, 103517 (2003). arXiv:hep-th/0307077

47. J. Martin, P. Peter, On the properties of the transition matrix in bouncing cosmologies. Phys. Rev. D 69, 107301 (2004). arXiv:hep-th/0403173

48. A. Nayeri, R.H. Brandenberger, C. Vafa, Producing a scaleinvariant spectrum of perturbations in a Hagedorn phase of string cosmology. Phys. Rev. Lett. 97, 021302 (2006). arXiv:hep-th/0511140

49. P. Peter, E.J. Pinho, N. Pinto-Neto, A non inflationary model with scale invariant cosmological perturbations. Phys. Rev. D 75, 023516 (2007). arXiv:hep-th/0610205

50. F. Finelli, P. Peter, N. Pinto-Neto, Spectra of primordial fluctuations in two-perfect-fluid regular bounces. Phys. Rev. D 77, 103508 (2008). arXiv:0709.3074

51. L.R. Abramo, P. Peter, K-Bounce. JCAP 0709, 001 (2007). arXiv:0705.2893

52. F.T. Falciano, M. Lilley, P. Peter, A classical bounce: constraints and consequences. Phys. Rev. D 77, 083513 (2008). arXiv:0802.1196

53. A. Linde, V. Mukhanov, A. Vikman, On adiabatic perturbations in the ekpyrotic scenario. JCAP 1002, 006 (2010). arXiv:0912.0944

54. L.R. Abramo, I. Yasuda, P. Peter, Non singular bounce in modified gravity. Phys. Rev. D 81, 023511 (2010). arXiv:0910.3422

55. R. Brandenberger, Matter bounce in Horava-Lifshitz cosmology. Phys. Rev. D 80, 043516 (2009). arXiv:0904.2835

56. R.H. Brandenberger, String gas cosmology: progress and problems. Class. Quantum Gravity 28, 204005 (2011). arXiv: 1105.3247

57. R.H. Brandenberger, The matter bounce alternative to inflationary cosmology. arXiv:1206.4196

58. Y.-F. Cai, D.A. Easson, R. Brandenberger, Towards a nonsingular bouncing cosmology. JCAP 1208, 020 (2012). arXiv:1206.2382

59. Y.-F. Cai, R. Brandenberger, P. Peter, Anisotropy in a nonsingular bounce. arXiv:1301.4703

60. K. Saaidi, H. Sheikhahmadi, A.H. Mohammadi, Interacting new agegraphic dark energy in a cyclic universe. Astrophys. Sp. Sci. 
338, 355 (2012). https://doi.org/10.1007/s10509-011-0944-y. arXiv: 1201.0275 [gr-qc]

61. N. Arkani-Hamed, J. Maldacena, Cosmological collider physics. arXiv:1503.08043 [hep-th]

62. E. Dimastrogiovanni, M. Fasiello, M. Kamionkowski, Imprints of massive primordial fields on large-scale structure. JCAP 1602, 017 (2016). arXiv: 1504.05993 [astro-ph.CO]

63. X. Chen, M.H. Namjoo, Y. Wang, Quantum primordial standard clocks. JCAP 1602(02), 013 (2016). arXiv:1509.03930 [astroph.CO]

64. X. Chen, M.H. Namjoo, Y. Wang, Probing the primordial universe using massive fields. Int. J. Mod. Phys. D 26(01), 1740004 (2016). arXiv:1601.06228 [hep-th]

65. H. Lee, D. Baumann, G.L. Pimentel, Non-gaussianity as a particle detector. JHEP 1612, 040 (2016). arXiv:1607.03735 [hep-th]

66. X. Chen, M.H. Namjoo, Y. Wang, A direct probe of the evolutionary history of the primordial universe. Sci. China Phys. Mech. Astron. 59(10), 101021 (2016). arXiv: 1608.01299 [astro-ph.CO]

67. P.D. Meerburg, M. Münchmeyer, J.B. Muñoz, X. Chen, Prospects for cosmological collider physics. JCAP 1703(03), 050 (2017). arXiv:1610.06559 [astro-ph.CO]

68. X. Chen, Y. Wang, Z.Z. Xianyu, Standard model background of the cosmological collider. Phys. Rev. Lett. 118(26), 261302 (2017). arXiv:1610.06597 [hep-th]

69. X. Chen, Y. Wang, Z.Z. Xianyu, Standard model mass spectrum in inflationary universe. JHEP 1704, 058 (2017). arXiv:1612.08122 [hep-th]

70. H. An, M. McAneny, A.K. Ridgway, M.B. Wise, Quasi single field inflation in the non-perturbative regime. arXiv:1706.09971 [hep-ph]

71. H. An, M. McAneny, A.K. Ridgway, M.B. Wise, Non-gaussian enhancements of galactic halo correlations in quasi-single field inflation. arXiv:1711.02667 [hep-ph]

72. A.V. Iyer, S. Pi, Y. Wang, Z. Wang, S. Zhou, Strongly coupled quasi-single field inflation. JCAP 1801(01), 041 (2018). arXiv: 1710.03054 [hep-th]

73. C. Armendariz-Picon, T. Damour, V.F. Mukhanov, k-inflation. Phys. Lett. B 458, 209 (1999). arXiv:hep-th/9904075

74. H. Sheikhahmadi, S. Ghorbani, K. Saaidi, Non-local scalar fields inflationary mechanism in light of Planck 2013. Astrophys. Sp. Sci. 357(2), 115 (2015). arXiv: 1502.05166

75. B.A. Bassett, S. Tsujikawa, D. Wands, Inflation dynamics and reheating. Rev. Mod. Phys. 78, 537 (2006). arXiv:astro-ph/0507632

76. D. Wands, Multiple field inflation. Lect. Notes Phys. 738, 275 (2008). arXiv:astro-ph/0702187 [ASTRO-PH]

77. R. Emami, Spectroscopy of masses and couplings during inflation. JCAP 1404, 031 (2014). arXiv:1311.0184 [hep-th]

78. H. Sheikhahmadi, E.N. Saridakis, A. Aghamohammadi, K. Saaidi, Hamilton-Jacobi formalism for inflation with nonminimal derivative coupling. JCAP 1610(10), 021 (2016). https:// doi.org/10.1088/1475-7516/2016/10/021 . arXiv:1603.03883 [gr-qc]

79. H. Sheikhahmadi, Schwinger-Keldysh mechanism in extended quasi single field inflation. Eur. Phys. J. C 79, 451 (2019). arXiv: 1901.01905 [gr-qc]

80. M. Alishahiha, E. Silverstein, D. Tong, DBI in the sky. Phys. Rev. D 70, 123505 (2004). arXiv:hep-th/0404084

81. D. Langlois, S. Renaux-Petel, D.A. Steer, T. Tanaka, Primordial perturbations and non-Gaussianities in DBI and general multifield inflation. Phys. Rev. D 78, 063523 (2008). arXiv:0806.0336

82. D. Langlois, S. Renaux-Petel, D.A. Steer, Multi-field DBI inflation: introducing bulk forms and revisiting the gravitational wave constraints. JCAP 0904, 021 (2009). arXiv:0902.2941

83. A. Golovnev, V. Mukhanov, V. Vanchurin, Vector inflation. JCAP 0806, 009 (2008). arXiv:0802.2068
84. P. Adshead, M. Wyman, Chromo-natural inflation: natural inflation on a steep potential with classical non-Abelian gauge fields. Phys. Rev. Lett. 108, 261302 (2012). arXiv:1202.2366

85. A. Maleknejad, M. Sheikh-Jabbari, Gauge-flation: inflation from non-Abelian gauge fields. arXiv: 1102.1513

86. A. Maleknejad, M. Sheikh-Jabbari, Non-Abelian gauge field inflation. Phys. Rev. D 84, 043515 (2011). arXiv:1102.1932

87. A. Maleknejad, M. Sheikh-Jabbari, J. Soda, Gauge fields and inflation. arXiv:1212.2921

88. A. Berera, Warm inflation. Phys. Rev. Lett. 75, 3218 (1995). $\quad$ https://doi.org/10.1103/PhysRevLett.75.3218. arXiv:astro-ph/9509049

89. A. Berera, Thermal properties of an inflationary universe. Phys. Rev. D 54, 2519 (1996). https://doi.org/10.1103/PhysRevD.54. 2519. arXiv:hep-th/9601134

90. A. Berera, L.Z. Fang, Thermally induced density perturbations in the inflation era. Phys. Rev. Lett. 74, 1912 (1995). https://doi.org/ 10.1103/PhysRevLett.74.1912. arXiv:astro-ph/9501024

91. A. Berera, Interpolating the stage of exponential expansion in the early universe: a possible alternative with no reheating. Phys. Rev. D 55, 3346 (1997). https://doi.org/10.1103/PhysRevD.55. 3346. arXiv:hep-ph/9612239

92. A. Berera, M. Gleiser, R.O. Ramos, Strong dissipative behavior in quantum field theory. Phys. Rev. D 58, 123508 (1998). https:// doi.org/10.1103/PhysRevD.58.123508. arXiv:hep-ph/9803394

93. A. Berera, Warm inflation at arbitrary adiabaticity: a model, an existence proof for inflationary dynamics in quantum field theory. Nucl. Phys. B 585, 666 (2000). https://doi.org/10.1016/ S0550-3213(00)00411-9. arXiv:hep-ph/9904409

94. M. Bastero-Gil, A. Berera, R.O. Ramos, J.G. Rosa, Warm little inflaton. Phys. Rev. Lett. 117(15), 151301 (2016). https://doi.org/ 10.1103/PhysRevLett.117.151301. arXiv:1604.08838 [hep-ph]

95. A. Berera, J. Mabillard, M. Pieroni, R.O. Ramos, Identifying universality in warm inflation. JCAP 1807(07), 021 (2018). https://doi.org/10.1088/1475-7516/2018/07/021. arXiv:1803.04982 [astro-ph.CO]

96. J. Yokoyama, A.D. Linde, Is warm inflation possible? Phys. Rev. D 60, 083509 (1999). arXiv:hep-ph/9809409

97. M. Bastero-Gil, A. Berera, R.O. Ramos, Dissipation coefficients from scalar and fermion quantum field interactions. JCAP 1109, 033 (2011). arXiv: 1008.1929

98. S. Bartrum, A. Berera, J.G. Rosa, Warming up for Planck. arXiv: 1303.3508

99. M. Naderi, A. Aghamohammadi, A. Refaei, H. Sheikhahmadi, The effects of low anisotropy on non-canonical scalar field with intermediate inflation. arXiv:1809.02348 [physics.gen-ph]

100. Z. Ghadiri, A. Refaei, A. Aghamohammadi, H. Sheikhahmadi, Constraints on warm power-law inflation in light of Planck 2013 and 2015 results. arXiv: 1809.00165 [gr-qc]

101. M.S. Turner, Coherent scalar field oscillations in an expanding universe. Phys. Rev. D 28, 1243 (1983)

102. L. Kofman, A.D. Linde, A.A. Starobinsky, Towards the theory of reheating after inflation. Phys. Rev. D 56, 3258-3295 (1997). arXiv:hep-ph/9704452

103. A. Mazumdar, J. Rocher, Particle physics models of inflation and curvaton scenarios. Phys. Rep. 497, 85-215 (2011). arXiv: 1001.0993

104. F. Finelli, R.H. Brandenberger, Parametric amplification of gravitational fluctuations during reheating. Phys. Rev. Lett. 82, 1362 1365 (1999). arXiv:hep-ph/9809490

105. B.A. Bassett, D.I. Kaiser, R. Maartens, General relativistic preheating after inflation. Phys. Lett. B 455, 84-89 (1999). arXiv:hep-ph/9808404

106. F. Finelli, R.H. Brandenberger, Parametric amplification of metric fluctuations during reheating in two field models. Phys. Rev. D 62, 083502 (2000). arXiv:hep-ph/0003172 
107. K. Jedamzik, M. Lemoine, J. Martin, Collapse of small-scale density perturbations during preheating in single field inflation. JCAP 1009, 034 (2010). arXiv:1002.3039

108. K. Jedamzik, M. Lemoine, J. Martin, Generation of gravitational waves during early structure formation between cosmic inflation and reheating. JCAP 1004, 021 (2010). arXiv:1002.3278

109. R. Easther, R. Flauger, J.B. Gilmore, Delayed reheating and the breakdown of coherent oscillations. JCAP 1104, 027 (2011). arXiv: 1003.3011

110. Planck Collaboration Collaboration, P. Ade et al., Planck 2013 results. I. Overview of products and scientific results. arXiv: 1303.5062

111. Planck Collaboration Collaboration, P. Ade et al., Planck 2013 results. XXII. Constraints on inflation. arXiv:1303.5082

112. Planck Collaboration Collaboration, P. Ade et al., Planck 2013 results. XXIV. Constraints on primordial non-Gaussianity. arXiv: 1303.5084

113. Supernova Search Team Collaboration, J.L. Tonry et al., Cosmological results from high-z supernovae. Astrophys. J. 594, 1-24 (2003). arXiv:astro-ph/0305008

114. Supernova Search Team Collaboration, A.G. Riess et al., Type Ia supernova discoveries at $r m z>1$ from the hubble space telescope: evidence for past deceleration and constraints on dark energy evolution. Astrophys. J. 607, 665-687 (2004). arXiv:astro-ph/0402512

115. A.G. Riess, L.-G. Strolger, S. Casertano, H.C. Ferguson, B. Mobasher et al., New hubble space telescope discoveries of type ia supernovae at $\mathrm{z}>1$ : narrowing constraints on the early behavior of dark energy. Astrophys. J. 659, 98-121 (2007). arXiv:astro-ph/0611572

116. A.G. Riess, L. Macri, S. Casertano, H. Lampeitl, H.C. Ferguson et al., A 3\% solution: determination of the hubble constant with the hubble space telescope and wide field camera 3. Astrophys. J. 730, 119 (2011). arXiv:1103.2976

117. SDSS Collaboration Collaboration, J.K. Adelman-McCarthy et al., The sixth data release of the sloan digital sky survey. Astrophys. J. Suppl. 175, 297-313 (2008). arXiv:0707.3413

118. SDSS Collaboration Collaboration, K.N. Abazajian et al., The seventh data release of the sloan digital sky survey. Astrophys. J. Suppl. 182, 543-558 (2009). arXiv:0812.0649

119. Euclid collaboration Collaboration, J. Amiaux et al., Euclid mission: building of a reference survey. arXiv:1209.2228

120. M.S. Turner, M.J. White, J.E. Lidsey, Tensor perturbations in inflationary models as a probe of cosmology. Phys. Rev. D 48 , 4613-4622 (1993). arXiv:astro-ph/9306029

121. M. Maggiore, Gravitational wave experiments and early universe cosmology. Phys. Rep. 331, 283-367 (2000). arXiv:gr-qc/9909001

122. H. Kudoh, A. Taruya, T. Hiramatsu, Y. Himemoto, Detecting a gravitational-wave background with next-generation space interferometers. Phys. Rev. D 73, 064006 (2006). arXiv:gr-qc/0511145

123. S. Kuroyanagi, C. Gordon, J. Silk, N. Sugiyama, Forecast constraints on inflation from combined CMB and gravitational wave direct detection experiments. Phys. Rev. D 81, 083524 (2010). arXiv:0912.3683

124. S. Kawamura, M. Ando, N. Seto, S. Sato, T. Nakamura et al., The Japanese space gravitational wave antenna: DECIGO. Class. Quantum Gravity 28, 094011 (2011)

125. P. Amaro-Seoane, S. Aoudia, S. Babak, P. Binetruy, E. Berti, et al., eLISA: astrophysics and cosmology in the millihertz regime. arXiv: 1201.3621

126. S. Kuroyanagi, C. Ringeval, T. Takahashi, Early universe tomography with CMB and gravitational waves. Phys. Rev. D 87, 083502 (2013). arXiv:1301.1778
127. J. Dunkley, E. Calabrese, J. Sievers, G. Addison, N. Battaglia, et al., The Atacama cosmology telescope: likelihood for smallscale CMB data. arXiv:1301.0776

128. J.L. Sievers, R.A. Hlozek, M.R. Nolta, V. Acquaviva, G.E. Addison, et al., The Atacama cosmology telescope: cosmological parameters from three seasons of data. arXiv:1301.0824

129. Z. Hou, C. Reichardt, K. Story, B. Follin, R. Keisler, et al., Constraints on cosmology from the cosmic microwave background power spectrum of the 2500-square degree SPT-SZ Survey. arXiv: 1212.6267

130. K. Story, C. Reichardt, Z. Hou, R. Keisler, K. Aird, et al., A measurement of the cosmic microwave background damping tail from the 2500-square-degree SPT-SZ survey. arXiv:1210.7231

131. CMBPol Study Team Collaboration, D. Baumann et al., CMBPol mission concept study: probing inflation with CMB polarization. AIP Conf .Proc. 1141, 10-120 (2009). arXiv:0811.3919

132. B. Crill, P. Ade, E. Battistelli, S. Benton, R. Bihary, et al., SPIDER: a balloon-borne large-scale CMB polarimeter. arXiv:0807.1548

133. M. Zaldarriaga, S.R. Furlanetto, L. Hernquist, 21 Centimeter fluctuations from cosmic gas at high redshifts. Astrophys. J. 608, 622-635 (2004). arXiv:astro-ph/0311514

134. A. Lewis, A. Challinor, The $21 \mathrm{~cm}$ angular-power spectrum from the dark ages. Phys. Rev. D 76, 083005 (2007). arXiv:astro-ph/0702600

135. M. Tegmark, M. Zaldarriaga, The Fast Fourier transform telescope. Phys. Rev. D 79, 083530 (2009). arXiv:0805.4414

136. V. Barger, Y. Gao, Y. Mao, D. Marfatia, Inflationary potential from $21 \mathrm{~cm}$ tomography and Planck. Phys. Lett. B 673, 173-178 (2009). arXiv:0810.3337

137. Y. Mao, M. Tegmark, M. McQuinn, M. Zaldarriaga, O. Zahn, How accurately can $21 \mathrm{~cm}$ tomography constrain cosmology? Phys. Rev. D 78, 023529 (2008). arXiv:0802.1710

138. P. Adshead, R. Easther, J. Pritchard, A. Loeb, Inflation and the scale dependent spectral index: prospects and strategies. JCAP 1102, 021 (2011). arXiv: 1007.3748

139. S. Clesse, L. Lopez-Honorez, C. Ringeval, H. Tashiro, M.H. Tytgat, Background reionization history from omniscopes. Phys. Rev. D 86, 123506 (2012). arXiv:1208.4277

140. J. Martin, C. Ringeval, First CMB constraints on the inflationary reheating temperature. Phys. Rev. D 82, 023511 (2010). arXiv: 1004.5525

141. H. Sheikhahmadi, A. Aghamohammadi, K. Saaidi, The effect of de Sitter like background on increasing the zero point budget of dark energy. Adv. High Energy Phys. 2016, 2594189 (2016). https://doi.org/10.1155/2016/2594189. arXiv:1407.0125 [gr-qc]

142. B. Abbott et al., LIGO Scientific Collaboration and Virgo Collaboration. Phys. Rev. Lett. 116, 061102 (2016)

143. A. Einstein, Sitzungsber. K. Preuss. Akad. Wiss. 1, 688 (1916)

144. B. Abbott et al., LIGO Scientific Collaboration and Virgo Collaboration. Phys. Rev. Lett. 116, 241103 (2016)

145. B. Abbott et al., LIGO Scientific Collaboration and Virgo Collaboration. Phys. Rev. Lett. 118, 221101 (2017)

146. B. Abbott et al., LIGO Scientific Collaboration and Virgo Collaboration. Phys. Rev. Lett. 119, 141101 (2017)

147. B. Abbott et al., LIGO Scientific Collaboration and Virgo Collaboration. Phys. Rev. Lett. 119, 161101 (2017)

148. B. Abbott et al. (LIGO Scientific Collaboration and Virgo Collaboration) (2017). arXiv: 1711.05578

149. A.N. Nitz et al., Astrophys. J 872, 2 (2019)

150. C. Corda, Interferometric detection of gravitational waves: the definitive test for General Relativity. Int. J. Mod. Phys. D 18, 2275 (2009). https://doi.org/10.1142/S0218271809015904. arXiv:0905.2502 [gr-qc]

151. C. Corda, The future of gravitational theories in the era of the gravitational wave astronomy. Int. J. Mod. Phys. D 27(05), 
1850060 (2018). https://doi.org/10.1142/S0218271818500608. arXiv: 1712.10318 [gr-qc]

152. C. Corda, Information on the inflaton field from the spectrum of relic gravitational waves. Gen. Rel. Grav. 42, 1323 (2010). Erratum: [Gen. Rel. Grav. 42, 1335 (2010)]. https://doi.org/10.1007/s10714-009-0895-6, https://doi.org/10. 1007/s10714-009-0917-4. arXiv:0909.4133 [gr-qc]

153. A.H. Guth, S.Y. Pi, The quantum mechanics of the scalar field in the new inflationary universe. Phys. Rev. D 32, 1899 (1985). https://doi.org/10.1103/PhysRevD.32.1899

154. D.H. Lyth, Large scale energy density perturbations and inflation. Phys. Rev. D 31, 1792 (1985). https://doi.org/10.1103/PhysRevD. 31.1792

155. J.J. Halliwell, Scalar fields in cosmology with an exponential potential. Phys. Lett. B 185, 341 (1987). https://doi.org/10.1016/ 0370-2693(87)91011-2

156. M. Sasaki, Large scale quantum fluctuations in the inflationary universe. Prog. Theor. Phys. 76, 1036 (1986). https://doi.org/10. 1143/PTP.76.1036

157. J.J. Halliwell, Decoherence in quantum cosmology. Phys. Rev. D 39, 2912 (1989). https://doi.org/10.1103/PhysRevD.39.2912

158. E. Calzetta, Anisotropy dissipation in quantum cosmology. Phys. Rev. D 43, 2498 (1991). https://doi.org/10.1103/PhysRevD.43. 2498

159. J.P. Paz, S. Sinha, Decoherence and back reaction in quantum cosmology: multidimensional minisuperspace examples. Phys. Rev. D 45, 2823 (1992). https://doi.org/10.1103/PhysRevD.45.2823

160. E. Calzetta, B.L. Hu, Noise and fluctuations in semiclassical gravity. Phys. Rev. D 49, 6636 (1994). https://doi.org/10.1103/ PhysRevD.49.6636. arXiv:gr-qc/9312036

161. A.L. Matacz, The coherent state representation of quantum fluctuations in the early universe. Phys. Rev. D 49, 788 (1994). https:// doi.org/10.1103/PhysRevD.49.788. arXiv:gr-qc/9212008

162. A. Berera, M. Gleiser, R.O. Ramos, A first principles warm inflation model that solves the cosmological horizon/flatness problems. Phys. Rev. Lett. 83, 264 (1999). https://doi.org/10.1103/ PhysRevLett.83.264. arXiv:hep-ph/9809583

163. R. Kubo, Statistical Mechanics: An Advanced Course with Problems and Solutions (North- Holland, Amsterdam, 1965)

164. R. Kubo, Statistical mechanical theory of irreversible processes. 1. General theory and simple applications in magnetic and conduction problems. J. Phys. Soc. Jpn. 12, 570 (1957). https://doi. org/10.1143/JPSJ.12.570

165. H.B. Callen, T.A. Welton, Irreversibility and generalized noise. Phys. Rev. 83, 34 (1951). https://doi.org/10.1103/PhysRev.83.34

166. A.O. Caldeira, A.J. Leggett, Influence of dissipation on quantum tunneling in macroscopic systems. Phys. Rev. Lett. 46, 211 (1981). https://doi.org/10.1103/PhysRevLett.46.211

167. D.F. Mota, J.D. Barrow, Varying alpha in a more realistic Universe. Phys. Lett. B 581, 141 (2004). https://doi.org/10.1016/j. physletb.2003.12.016. arXiv:astro-ph/0306047

168. J. Khoury, A. Weltman, Chameleon fields: awaiting surprises for tests of gravity in space. Phys. Rev. Lett. 93, 171104 (2004). https://doi.org/10.1103/PhysRevLett.93.171104. arXiv:astro-ph/0309300

169. J. Khoury, A. Weltman, Chameleon cosmology. Phys. Rev. D 69, 044026 (2004). https://doi.org/10.1103/PhysRevD.69. 044026. arXiv:astro-ph/0309411

170. P. Brax, C. van de Bruck, A.C. Davis, J. Khoury, A. Weltman, Chameleon dark energy. AIP Conf. Proc. 736, 105 (2004). https:// doi.org/10.1063/1.1835177. arXiv:astro-ph/0410103

171. J. Khoury, Chameleon field theories. Class. Quantum Gravity 30, 214004 (2013). https://doi.org/10.1088/0264-9381/30/21/ 214004. arXiv:1306.4326 [astro-ph.CO]

172. T.P. Waterhouse, An introduction to chameleon gravity. arxiv:astro-ph/0611816
173. T. Clifton, J.D. Barrow, Decaying gravity. Phys. Rev. D 73, 104022 (2006). https://doi.org/10.1103/PhysRevD.73.104022. arXiv:gr-qc/0603116

174. S. Das, N. Banerjee, Brans-Dicke scalar field as a chameleon. Phys. Rev. D 78, 043512 (2008). https://doi.org/10.1103/ PhysRevD.78.043512. arXiv:0803.3936 [gr-qc]

175. K. Saaidi, A. Mohammadi, H. Sheikhahmadi, $\gamma$ parameter and solar system constraint in chameleon Brans Dick theory. Phys. Rev. D 83, 104019 (2011). https://doi.org/10.1103/PhysRevD.83. 104019. arXiv:1201.0271 [gr-qc]

176. K. Saaidi, A. Mohammadi, T. Golanbari, H. Sheikhahmadi, B. Ratra, Quark-Hadron phase transition for a chameleon BransDicke model of Brane gravity. Phys. Rev. D 86, 045007 (2012). https://doi.org/10.1103/PhysRevD.86.045007. arXiv:1201.0372 [gr-qc]

177. K. Saaidi, H. Sheikhahmadi, J. Afzali, Chameleon mechanism with a new potential. Astrophys. Sp. Sci. 333, 501 (2011). https://doi.org/10.1007/s10509-011-0675-0. arXiv:1011.0075 [physics.gen-ph]

178. H. Farajollahi, A. Salehi, Attractors, statefinders and observational measurement for chameleonic Brans-Dicke cosmology. JCAP 1011, 006 (2010). https://doi.org/10.1088/1475-7516/ 2010/11/006. arXiv:1010.3589 [gr-qc]

179. H. Farajollahi, A. Salehi, F. Tayebi, A. Ravanpak, Stability analysis in tachyonic potential chameleon cosmology. JCAP 1105, 017 (2011). https://doi.org/10.1088/1475-7516/2011/05/ 017. arXiv: 1105.4045 [gr-qc]

180. D.F. Mota, C.A.O. Schelpe, Evolution of the Chameleon scalar field in the early universe. Phys. Rev. D 86, 123002 (2012). https:// doi.org/10.1103/PhysRevD.86.123002. arXiv:1108.0892 [astroph.CO]

181. K. Hinterbichler, J. Khoury, H. Nastase, R. Rosenfeld, Chameleonic inflation. JHEP 1308, 053 (2013). https://doi.org/ 10.1007/JHEP08(2013)053. arXiv:1301.6756 [hep-th]

182. P. Creminelli, J. Gleyzes, L. Hui, M. Simonović, F. Vernizzi, Single-field consistency relations of large scale structure. Part III: test of the equivalence principle. JCAP 1406, 009 (2014). https://doi.org/10.1088/1475-7516/2014/06/009. arXiv:1312.6074 [astro-ph.CO]

183. N. Saba, M. Farhoudi, Chameleon field dynamics during inflation. Int. J. Mod. Phys. D 27(04), 1850041 (2017). https://doi.org/10. 1142/S0218271818500414. arXiv:1711.09682 [gr-qc]

184. S.M. Carroll, Quintessence and the rest of the world. Phys. Rev. Lett. 81, 3067 (1998). https://doi.org/10.1103/PhysRevLett.81. 3067. arXiv:astro-ph/9806099

185. T. Damour, A.M. Polyakov, The string dilaton and a least coupling principle. Nucl. Phys. B 423, 532 (1994). https://doi.org/10.1016/ 0550-3213(94)90143-0. arXiv:hep-th/9401069

186. J.D. Brown, Action functionals for relativistic perfect fluids. Class. Quantum Gravity 10, 1579 (1993). https://doi.org/10.1088/ 0264-9381/10/8/017. arXiv:gr-qc/9304026

187. J.D. Brown, J.W. York Jr., The microcanonical functional integral. 1. The gravitational field. Phys. Rev. D 47, 1420 (1993). https:// doi.org/10.1103/PhysRevD.47.1420. arXiv:gr-qc/9209014

188. T.P. Sotiriou, V. Faraoni, Modified gravity with R-matter couplings and (non-)geodesic motion. Class. Quantum Gravity 25, 205002 (2008). https://doi.org/10.1088/0264-9381/25/20/ 205002. arXiv:0805.1249 [gr-qc]

189. K. Saaidi, H. Sheikhahmadi, T. Golanbari, S.W. Rabiei, On the holographic dark energy in chameleon scalar-tensor cosmology. Astrophys. Sp. Sci. 348, 233 (2013). https://doi.org/10.1007/ s10509-013-1491-5. arXiv:1404.2139 [gr-qc]

190. A. Aghamohammadi, K. Saaidi, A. Mohammadi, H. Sheikhahmadi, T. Golanbari, S.W. Rabiei, Effect of an external interaction mechanism in solving agegraphic dark energy problems. 
Astrophys. Sp. Sci. 345(1), 17 (2013). https://doi.org/10.1007/ s10509-013-1386-5. arXiv:1402.2608 [physics.gen-ph]

191. H. Sheikhahmadi, Comments on "Cosmic evolution in BransDicke chameleon cosmology". Eur. Phys. J. Plus 133, 366 (2018). https://doi.org/10.1140/epjp/i2018-12235-3. arXiv:1802.06358 [gr-qc]
192. K. Saaidi, (Non-) geodesic motion in chameleon Brans Dicke model. Astrophys. Sp. Sci. 345, 431 (2013). https://doi.org/10. 1007/s10509-013-1407-4. arXiv:1205.3542 [gr-qc] 